\title{
A RELAXATION MODEL FOR LIQUID-VAPOR PHASE CHANGE WITH METASTABILITY*
}

\author{
FRANÇOIS JAMES ${ }^{\dagger}$ AND HÉLÈNE MATHIS ${ }^{\ddagger}$
}

\begin{abstract}
We propose a model that describes phase transition including metastable states present in the van der Waals equation of state. From a convex optimization problem on the Helmholtz free energy of a mixture, we deduce a dynamical system that is able to depict the mass transfer between two phases, for which equilibrium states are either metastable states, stable states or a coexistent state. The dynamical system is then used as a relaxation source term in an isothermal $4 \times 4$ two-phase model. We use a finite volume scheme that treats the convective part and the source term in a fractional step way. Numerical results illustrate the ability of the model to capture phase transition and metastable states.
\end{abstract}

Key words. Thermodynamics of phase transition, metastable states, nonlinear hyperbolic system with relaxation, van der Waals equation of state.

AMS subject classifications. 35Q79, 35L40, 76T10, 37N10, 80A10.

\section{Introduction}

In the last decades considerable research has been devoted to the simulation of liquid-vapor phase change, which is of major importance in several industrial applications. For instance liquid-vapor flows are present in water circuit of pressurized water reactors in which the water can be submitted to saturation pressure and temperature $[11,12]$. The phenomena we are interested in are complex phase changes including the possible appearance of metastable states. An example is the metastable vapor which is a gaseous state where the pressure is higher than the saturation pressure. Such states are very unstable and a very small perturbation will bring out a droplet of liquid inside of the gas [25]. This phenomenon can appear at saturated pressure (or at saturated temperature for metastable liquid). It is the case in pressurized water reactor during a loss of coolant accident when sudden vaporization occurs due to the drop of pressure inside the superheated liquid [21].

In this paper, we focus on situations where the heterogeneity of the fluid and the thermodynamic conditions allow the diphasic flow to be described by a compressible averaged model using Euler type equations. Other models can be considered, which account for very smale scale by means of Korteweg type tensors including dispersive and dissipative effects. Such models allow to preserve metastable states but give only a microscopic description of the flow, see $[1,6,22,27,33,34]$ and the references cited herein. In the averaged models framework, one can distinguish between one-fluid and two-fluid models.

The one-fluid model approach consists in describing the fluid flow as a single substance that can be present in its vapor or its liquid phase. Assuming that the thermodynamic equilibrium is reached instantaneously (quasi-static process), then the Euler system has to be closed by an equation of state (EoS) able to depict either the pure phases (liquid or vapor) and the phase transition. A typical example is the van der

\footnotetext{
* Received: July 20, 2015; accepted (in revised form): March 10, 2016. Communicated by Francois Bouchut.

†Mathématiques - Analyse, Probabilités, Modélisation - Orléans (MAPMO), Université d'Orléans \& CNRS UMR 7349, BP 6759, F-45067 Orléans Cedex 2, France (francois.james@univ-orleans.fr).

${ }^{\ddagger}$ Laboratoire Jean Leray, Université de Nantes \& CNRS UMR 6629, BP 92208, F-44322 Nantes Cedex 3, France (helene.mathis@univ-nantes.fr).
} 
Waals EoS, which is well-known to depict stable and metastable states below the critical temperature. However this EoS is not valid in the so-called spinodal zone where the pressure is a decreasing function of the density. This forbids the use of instantaneous kinetic exchanges, since the pressure is always given by the EoS, and a decreasing pressure leads to a loss of hyperbolicity in Euler equations, hence to instabilities and computational failure.

To overcome this defect and recover that the phase transition happens at constant pressure, temperature, and chemical potential, the van der Waals pressure is commonly corrected by the Maxwell equal area rule [8]. This construction leads to a correct constant pressure in the spinodal zone but removes the metastable regions.

Another way to provide a unique EoS able to depict pure phases and phase transition has been studied in $[2,14,17,24,28]$. It consists in considering that each phase is depicted by its own convex energy (that is its own monotone pressure law). According to the second principle of thermodynamics, the mixture equilibrium energy corresponds to the inf-convolution of the partial energies. As a result the mixture equilibrium energy coincides with the convex hull of the minimum of the two partial energies. The resulting pressure law of the mixture turns out to be composed of the monotone branches of the liquid and vapor pressure laws and is constant in the phase transition zone. Hence, it is clear that such a construction prevents the appearance of metastable phases.

Still in the context of one-fluid models, it is also possible to drop out the assumption of instantaneous equilibrium. The model involves then relaxation terms, which can be of various forms, but are the expression of a pulling back force to the equilibrium. We have to consider extended versions of the Euler system, which is supplemented by partial differential equations on additional quantities such as the volume fraction of the vapor phase or partial masses. This approach has been used in $[5,18,26,30,32]$ and in $[10,15]$ in the isothermal case. In the later references the question of preservation of metastable states is addressed.

We mention briefly another way to describe diphasic flows, which consists in considering a two-fluid approach to model liquid-vapor phase change. Initially developed to depict the motion of multicomponent flows [4], such a modeling assumes that the fluid can locally be present under both phase. Hence the model admits two pressures, two velocities and two temperatures and is supplemented by additional equations on the volume fraction. Phase transition is achieved by chemical and mechanical relaxation processes, in the limit where the kinetics is considered infinitely fast, see for instance $[30,32,35]$.

One of the present drawbacks of the averaged models (one or two-fluid) with relaxation is that there is no global agreement on the equations satisfied by the fractions and on the transfer terms [13]. Moreover the preservation of the metastable states seems to be out of reach in this framework.

In this paper, we intend to provide a model able to depict liquid-vapor phase change and metastable states of a single component, say some liquid in interaction with its own vapor. We focus on a one-fluid description of the motion while the phase transition is driven by transfer terms that will be coupled to fluid equations through a finite relaxation speed.

The modelling of the phase transition is the core of this work. For the sake of simplicity we assume the system to be isothermal. We propose transfer terms obtained through the minimization of the Helmholtz free-energy of the two-phase system. We use for both phases the same equation of state which has to be non monotone, typically the reduced van der Waals equation. This choice allows us to recover all possible equilibria: 
pure phases (liquid or vapor), metastable states, and coexistence states characterized by the equality of pressures and chemical potentials. These are physically admissible states, but the set of equilibrium states also contains the spinodal zone, which is irrelevant. Thus the key point now is to characterize the physical stability, and hence admissibility, of these states. It turns out that this has to be done in terms of their dynamical behaviour. More precisely, we design a dynamical system which is able to depict all the stable equilibria of the system as attraction points. In particular we show that metastable states and mixtures have different basins of attraction, so that they can be differentiated only by their long-time behaviour with respect to initial conditions. Hence there is no hope to recover both metastable states and coexistent states under the assumption of instantaneous equilibrium, which amounts precisely to choose a priori this long-time behaviour.

This dynamical system is used as a transfer term in an isothermal two-phase model in the spirit of [29] and [3]. The extended Euler system we obtain in this way provides a regularization of the isothermal Euler equation with van der Waals EoS, which takes the form of a mixture zone surrounding the physical interfaces, see Section 5 below.

The paper is organized as follows. Section 2 is devoted to the thermodynamics of a two-phase fluid. We provide the definitions of the common potentials and give some details on the reduced van der Waals model. Assuming that both phases follow the same non monotone EoS, we describe the thermodynamic equilibrium as the result of a minimization process on the Helmholtz free energy of the two-phase fluid. The section ends with the study of the equilibria of the optimality system. Section 3 is the core of this work. It is devoted to the construction of the dynamical system based on the results of the previous section. A few numerical simulations illustrate the ability of the system to catch both the Maxwell line and the metastable states in the van der Waals EoS. The dynamical system is then plugged as a source term in a $4 \times$ 4 isothermal model in Section 4. We provide a study of the homogeneous system, which is conditionally hyperbolic. However we prove that for smooth solutions the hyperbolicity regions are invariant domains of the system with relaxation. In Section 5 we present several numerical illustrations which assess the ability of the model to deal with metastable states. They are obtained using a classical finite volume schemes that treats the convective part and the source terms with a time-splitting technique.

\section{Thermodynamics and the van der Waals EoS}

2.1. Thermodynamics of a single fluid. Consider a fluid of mass $M \geq 0$ occupying a volume $V \geq 0$, assumed to be at constant temperature $T$. If the fluid is homogeneous and at rest, its behavior is entirely described by its mass, its volume and its Helmholtz free-energy F. According to Gibbs' formalism [16], the fluid is at equilibrium if its Helmholtz free-energy is a function, also denoted by $F$, of its mass $M$, and volume $V$

$$
F:(M, V) \rightarrow F(M, V) .
$$

Notice that we do not address yet the stability of equilibrium states. At this level the involved quantities are extensive, which means that they share the same scaling as the volume $V$. This corresponds to the notion of homogeneous sample: for twice the volume, the mass is double, and the energy, as well. The mathematical consequence of this notion is that extensive variables have to be related through positively homogeneous functions of degree 1 (PH1), namely

$$
\forall \lambda>0, \quad F(\lambda M, \lambda V)=\lambda F(M, V) .
$$


We assume in addition, and without loss of generality, that the energy function $F$ belongs to $\mathcal{C}^{2}\left(\mathbb{R}^{+} \times \mathbb{R}^{+}\right)$.

REMARK 2.1. The regularity of $F$ seems to preclude phase transitions, but this is not the case because no convexity assumption is made at this stage. We shall come back to this in more details in the next section.

We now introduce intensive quantities, by which we mean that they do not depend on the volume scaling. A typical example is the density $\rho=M / V$, but such quantities also appear as derivatives of the equilibrium relation (2.2), which are homogeneous of degree 0 by construction. In this way two fundamental quantities are to be considered, namely the pressure $p$ and the chemical potential $\mu$, defined by

$$
p=-\frac{\partial F}{\partial V}(M, V), \quad \mu=\frac{\partial F}{\partial M}(M, V) .
$$

Notice that these quantities are defined only when the system is at equilibrium, and we recover the classical thermodynamic relation for isothermal flows

$$
d F=-p d V+\mu d M
$$

Another classical property of thermodynamic potentials is the so-called Gibbs' relation, which results from the Euler relation for PH1 functions

$$
F(M, V)=\nabla F(M, V) \cdot\left(\begin{array}{c}
M \\
V
\end{array}\right)
$$

Using Equation (2.3), we obtain

$$
F(M, V)=\mu M-p V
$$

For most of the following computations it is useful to rewrite the preceding relations using intensive variables. For a fixed volume $V$, we denote $f$ the Helmholtz free-energy per unit volume, which is a function of the density $\rho=M / V$

$$
f(\rho)=f\left(\frac{M}{V}\right)=\frac{1}{V} F(M, V)=F\left(\frac{M}{V}, 1\right) .
$$

We keep the notations $p$ and $\mu$ the pressure and the chemical potential as functions of the density $\rho$ :

$$
p(\rho)=p\left(\frac{M}{V}\right)=-\frac{\partial F}{\partial V}\left(\frac{M}{V}, 1\right), \quad \mu(\rho)=\mu\left(\frac{M}{V}\right)=\frac{\partial F}{\partial M}\left(\frac{M}{V}, 1\right) .
$$

Thus we obtain an intensive form of the Gibbs' relation (2.5)

$$
f(\rho)=\rho \mu(\rho)-p(\rho) .
$$

On the other hand, from the definitions of $p, \mu$, and $f$ we obtain easily the following relations

$$
\mu(\rho)=f^{\prime}(\rho), \quad p(\rho)=\rho f^{\prime}(\rho)-f(\rho), \quad \rho \mu^{\prime}(\rho)=p^{\prime}(\rho) .
$$


2.2. Example: the van der Waals EoS. The extensive energy of a van der Waals (monoatomic) fluid is

$$
F(M, V)=-\frac{a M^{2}}{V}+R T\left(M \log \frac{M}{V-M b}-M\right)
$$

where $R$ stands for the perfect gases constant and $a$ and $b$ are positive constants $(a=b=0$ leads to the perfect gases law). Since we consider the isothermal model, the temperature $T$ is a parameter here. We refer to [25, Ch. 7] for a justification of this law from statistical thermodynamics. The constant $b$ is proportional to the proper volume of a particle such that $V>M b$, and the potential $\frac{a}{V}$ depicts the interaction between particles.

The pressure and the chemical potential associated read

$$
\begin{aligned}
& p\left(\frac{M}{V}\right)=-\frac{a M^{2}}{V^{2}}+R T \frac{M}{V-M b}, \\
& \mu\left(\frac{M}{V}\right)=-2 \frac{a M}{V}+R T \log \frac{M}{V-M b}+R T \frac{M b}{V-M b} .
\end{aligned}
$$

The intensive quantities are

$$
\begin{aligned}
& f(\rho)=-a \rho^{2}+R T \rho\left(\log \left(\frac{\rho}{1-b \rho}\right)-1\right), \\
& p(\rho)=-a \rho^{2}+\frac{\rho R T}{1-b \rho}, \\
& \mu(\rho)=-2 a \rho+R T \log \frac{\rho}{1-b \rho}+R T \frac{b \rho}{1-b \rho} .
\end{aligned}
$$

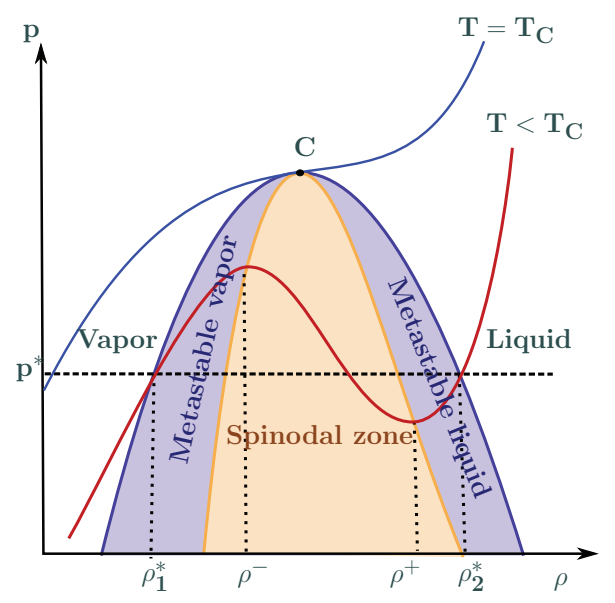

FIG. 2.1. Phase diagram for the van der Waals EoS in the $(p, \rho)$ plane. Below the critical temperature $T_{C}$, the isotherm curve decreases in the spinodal zone which is delimited by the densities $\rho^{-}<\rho^{+}$. In that area the isotherm is commonly replaced by an horizontal segment (dashed line) which coincides with the isobaric line at constant pressure $p=p^{*}$. Such a construction defines the two densities $\rho_{1}^{*}$ and $\rho_{2}^{*}$.

The behavior of the isotherm curves in the pressure-density plane is depicted in Figure 2.1. The critical temperature $T_{c}$ is the lower limit of temperatures such that the 
pressure is an increasing function of the density. At $T=T_{c}$ the pressure curve admits an unique horizontal inflection point, called the critical point, denoted $C$ on Figure 2.1.

In the sequel we will consider the reduced form of the van der Waals equation (see $\left[25\right.$, Ch. 8]). Denoting $\left(\rho_{c}, p_{c}\right)$ the coordinates of the critical point one has

$$
\begin{aligned}
& p^{\prime}\left(\rho_{c}\right)=-2 a \rho_{c}+\frac{R T_{C}}{\left(1-b \rho_{c}\right)^{2}}=0, \\
& p^{\prime \prime}\left(\rho_{c}\right)=-2 a+\frac{2 b R T_{c}}{\left(1-b \rho_{c}\right)^{3}}=0 .
\end{aligned}
$$

Considering normalized critical quantities, that is setting $\rho_{c}=1, p_{c}=1$, and $T_{c}=1$, one obtains the reduced van der Waals EOS with $R=8 / 3, a=3$, and $b=1 / 3$ and

$$
\begin{aligned}
& f(\rho)=-3 \rho^{2}+\frac{8}{3} \rho(\log (3 \rho /(3-\rho))-1) \\
& p(\rho)=-3 \rho^{2}+\frac{8 \rho}{3-\rho} \\
& \mu(\rho)=-6 \rho+\frac{8}{3} \log (3 \rho /(3-\rho))+\frac{8}{3}(3 \rho /(3-\rho))
\end{aligned}
$$

for $0<\rho<3$.

Below the critical temperature $T_{c}$ the pressure is not monotone with respect to the density (see the red curve on Figure 2.1): in a region called spinodal zone, delimited for a given temperature by the densities $\rho^{-}<\rho^{+}$, the pressure decreases with respect to the density and thus leading to unstable states. According to the relations (2.9) the energy $f$ is non-convex in the spinodal zone.

In that region the isotherm is commonly replaced by the Maxwell area rule in order to recover that phase transition happens at constant pressure and constant chemical potential. The Maxwell construction is commonly applied on the pressure (see [8] for instance) in such a way that the two zones delimited by the van der Waals isotherm and the Maxwell line (above and below the Maxwell line respectively) have the same area. This is not the case in Figure 2.1 because in our context, the equal area rule is obtained on the chemical potential, see Section 2.4 below (Proposition 2.1). In any case, the idea is that the isotherm curve is replaced locally by the horizontal segment, the so-called Maxwell line, that coincides with some isobaric line $p=p^{*}$. Such a construction defines two densities, denoted $\rho_{1}^{*}$ and $\rho_{2}^{*}$, as well as the value of $p^{*}$. An equivalent way to compute this correction is to build the convex hull of the function $f$, see [2] and [17].

However this construction removes the admissible regions delimited by the densities where the pressure law is still nondecreasing. Such regions are called metastable regions. At a given temperature these regions correspond to densities belonging to the range $\left[\rho_{1}^{*}, \rho^{-}\right]$and $\left[\rho^{+}, \rho_{2}^{*}\right]$.

2.3. Thermodynamics of phase transition. The van der Waals model depicts the thermodynamic behavior of a pure substance under its liquid state, gaseous state and the coexistence state. The non-convexity of the EoS allows to capture metastable states but does not give a relevant representation of the coexistence phase. A convenient way to cope with this problem is to represent the fluid under consideration as a set of several copies of itself under different pure phases (liquid or gaseous phases). Such a representation is used in $[2,5,14,17,18,24,26,30,32]$ assuming that the fluid is described by two copies, each one satisfying a convex EoS that differs from the one of another copy. See also [23] where such a representation is used in the context of adsorption-desorption of a mixture. 
We adopt here a slightly modified approach. Let us consider $I$ copies of the pure substance, $I \geq 1$ being some a priori arbitrary integer. Each copy is depicted by its mass $M_{i} \geq 0$ and its volume $V_{i}>0$, and is assumed to be at equilibrium. Hence a copy can occupy a volume with zero mass. We suppose that each copy follows the same nonconvex energy function $F\left(M_{i}, V_{i}\right)$, typically the van der Waals extensive energy given by Equation (2.10). This assumption contrasts with the aforementioned references where different convex energy functions are considered, and the number of copies is prescribed.

Thanks to the mass conservation, the complete system has a total mass $M=$ $\sum_{i=1}^{I} M_{i}$. Assuming that the copies are immiscible, the total volume is $V=\sum_{i=1}^{I} V_{i}$ (for a mixture of gas, one has $V_{i}=V, \forall i=1, \ldots, I$, and this condition implies Dalton's law, see [8]). Out of thermodynamic equilibrium the free Helmholtz energy of the system reads

$$
\mathcal{F}\left(\left(M_{i}, V_{i}\right)_{i}\right)=\sum_{i=1}^{I} F\left(M_{i}, V_{i}\right) .
$$

Let us fix the total mass $M$ and volume $V$ of the system. According to the second principle of thermodynamics (see [8] for instance), the thermodynamic equilibrium corresponds to the solutions of the constrained optimization problem

$$
\inf _{I \geq 1, M_{i} \geq 0, V_{i} \geq 0}\left\{\sum_{i=1}^{I} F\left(M_{i}, V_{i}\right) ; \sum_{i=1}^{I} V_{i}=V, \sum_{i=1}^{I} M_{i}=M\right\} .
$$

We stress the fact that the total number $I$ of possible copies is not a priori fixed here. However, as a consequence of Carathéodory's theorem in dimension 2 (see e.g. [31, Ch. $17]$ ), we can state the Gibbs' phase rule (see [25, Ch. 9]), which gives the expected result in this context.

LEMma 2.1 (Gibbs' phase rule). We have $I \leq 2$.

In the sequel we use just as well the term phase as the term copy.

Taking into account Lemma 2.1 and the above intensive formulations, we rewrite the constrained optimization problem for a fixed mass $M$ and volume $V$, hence for a fixed $\rho$, as

$$
\inf _{\substack{\rho_{1} \geq 0 \\ \rho_{2} \geq 0}}\left\{\alpha_{1} f\left(\rho_{1}\right)+\alpha_{2} f\left(\rho_{2}\right)\right\}
$$

under the constraints

$$
\begin{aligned}
\alpha_{1}+\alpha_{2} & =1, \\
\alpha_{1} \rho_{1}+\alpha_{2} \rho_{2} & =\rho .
\end{aligned}
$$

Here $\alpha_{i}=\frac{V_{i}}{V} \in[0,1]$ denotes the volume fraction and $\rho_{i}=\frac{M_{i}}{V_{i}} \geq 0$ the density of the phase $i=1,2$. Notice that we removed the optimization on the phase number $I$, since single phases can be recovered by $\rho_{1}=\rho_{2}$ (and any $\alpha_{i}$ ) or one $\alpha_{i}=0$ (with undetermined $\rho_{j}$, $j \neq i)$.

We rule out the equality case by noticing that, provided $\rho_{1} \neq \rho_{2}$, we can rewrite the constraints $(2.15)-(2.16)$ as

$$
\begin{array}{rlrl}
\alpha_{1}: \mathbb{R}^{+} \times \mathbb{R}^{+} \times \mathbb{R}^{+} & \rightarrow[0,1] & \alpha_{2}: \mathbb{R}^{+} \times \mathbb{R}^{+} \times \mathbb{R}^{+} \rightarrow[0,1] \\
\left(\rho, \rho_{1}, \rho_{2}\right) & \mapsto \frac{\rho-\rho_{2}}{\rho_{1}-\rho_{2}}, & \left(\rho, \rho_{1}, \rho_{2}\right) & \mapsto-\frac{\rho-\rho_{1}}{\rho_{1}-\rho_{2}} .
\end{array}
$$


We have $\alpha_{1} \geq 0$ if and only if $\rho_{1} \leq \rho \leq \rho_{2}$ or $\rho_{1} \geq \rho \geq \rho_{2}$. Therefore, accounting on the reduced form of the van der Waals model (2.13), we shall assume in the sequel that the densities $\rho, \rho_{1}$, and $\rho_{2}$ satisfy

$$
0<\rho_{1} \leq \rho \leq \rho_{2}<3 \quad \text { and } \quad \rho_{1}<\rho_{2} .
$$

This is not a restriction, as we shall see below (see Proposition 3.1).

One can also introduce the mass fraction $\varphi_{i}=\frac{M_{i}}{M}, i=1,2$ defined by

$$
\begin{aligned}
\varphi_{1}: \mathbb{R}^{+} \times \mathbb{R}^{+} \times \mathbb{R}^{+} \rightarrow[0,1] & \varphi_{2}: \mathbb{R}^{+} \times \mathbb{R}^{+} \times \mathbb{R}^{+} \rightarrow[0,1] \\
\left(\rho, \rho_{1}, \rho_{2}\right) \mapsto \frac{\frac{1}{\rho}-\frac{1}{\rho_{2}}}{\frac{1}{\rho_{1}}-\frac{1}{\rho_{2}}}, & \left(\rho, \rho_{1}, \rho_{2}\right) \mapsto \frac{\frac{1}{\rho}-\frac{1}{\rho_{1}}}{\frac{1}{\rho_{2}}-\frac{1}{\rho_{1}}},
\end{aligned}
$$

that satisfy $\varphi_{1}+\varphi_{2}=1$ and $\varphi_{1} \varphi_{2} \geq 0$ if and only if the assumption $\left(H_{1}\right)$ is satisfied. Such quantities will be useful in the mathematical study of the isothermal two-phase flow model introduced in Section 4.

REMARK 2.2. In the aforementioned references $[2,5,14,17,18,24]$ the method consists in describing the two-phase fluid by a coexistence of two copies of the same substance. The description can be made either on the extensive variables or the intensive one. Unlike our present approach the two copies do not follow the same EoS: each copy is described by its own strictly convex extensive energy $F_{i}, i=1,2$, which is a function of the mass $M_{i}$ and the volume $V_{i}$ of the phase. Following the second principle of thermodynamics [8], at equilibrium, the extensive energy of the two-phase fluid is given by

$$
F((M, V)):=F_{1} \square F_{2}(M, V)=\min _{V_{1} \geq 0, M_{1} \geq 0} F_{1}\left(M_{1}, V_{1}\right)+F_{2}\left(M-M_{1}, V-V_{1}\right),
$$

under the constraints of mass conservation $M=M_{1}+M_{2}$ and immiscibility (without vacuum) $V=V_{1}+V_{2}$. This operation $\square$ is called inf-convolution operation in the convex analysis framework [19]. In [17] the authors investigate the link between the infconvolution, the Legendre transform and the $(\max ,+)$ algebra. The Legendre transform of a energy $F$ is a convex function $\left(M^{*}, V^{*}\right) \rightarrow F^{*}\left(M^{*}, V^{*}\right)$ defined by

$$
F^{*}\left(M^{*}, V^{*}\right)=\inf _{M^{*} \geq 0, V^{*} \geq 0}\left\{M^{*} M+V^{*} V-F(M, V)\right\} .
$$

The inf-convolution is transformed into an addition by the Legendre transform which implies that

$$
\left(F_{1} \square F_{2}\right)^{*}=F_{1}^{*}+F_{2}^{*} .
$$

Moreover in the case of convex lower semi continuous (slc) functions $F_{i}, i=1,2$, one has

$$
F_{1} \square F_{2}=\left(F_{1} \square F_{2}\right)^{* *}=\left(F_{1}^{*}+F_{2}^{*}\right)^{*} .
$$

It means that the energy $F_{e q}$ of the two-phase fluid at equilibrium is given by

$$
F_{e q}=F^{* *},
$$

where $F^{* *}$ is the convex hull of the energy $F$. As it is proved in [2] the construction of the convex hull of the energy $F$ is equivalent to the Maxwell construction. Hence the operation (2.20) removes the metastable regions. 
2.4. Equilibrium states. This section is devoted to the characterization of the equilibrium states of the thermodynamic system, that is states that realize the infimum in the optimization problem (2.14)-(2.16). The function we minimize is defined by:

$$
\begin{aligned}
\tilde{\mathcal{F}}:[0,1] \times[0,1] \times \mathbb{R}^{+} \times \mathbb{R}^{+} & \rightarrow \mathbb{R} \\
\left(\alpha_{1}, \alpha_{2}, \rho_{1}, \rho_{2}\right) & \mapsto \alpha_{1} f\left(\rho_{1}\right)+\alpha_{2} f\left(\rho_{2}\right),
\end{aligned}
$$

and is $C^{1}$ on the space $\left\{\rho_{i} \geq 0, i=1,2\right\}$. The constraints (2.15)-(2.16) are affine, so they are also $C^{1}$. We are thus in position to use the Lagrange multipliers characterization of the infimum (see [31, Ch. 28]): $\lambda_{\alpha} \in \mathbb{R}$ and $\lambda_{\rho} \in \mathbb{R}$ respectively correspond to the constraints (2.15) and (2.16).

Using the definition (2.8) of the free-energy $f$ and the pressure and chemical potential definitions (2.9), one deduces the following optimality system of equations

$$
\begin{aligned}
f\left(\rho_{1}\right)+\lambda_{\alpha}+\lambda_{\rho} \rho_{1} & =0, \\
f\left(\rho_{2}\right)+\lambda_{\alpha}+\lambda_{\rho} \rho_{2} & =0, \\
\alpha_{1} \mu\left(\rho_{1}\right)+\lambda_{\rho} \alpha_{1} & =0, \\
\alpha_{2} \mu\left(\rho_{2}\right)+\lambda_{\rho} \alpha_{2} & =0 .
\end{aligned}
$$

From this optimality system we deduce immediately that there are two different kinds of equilibria.

Lemma 2.2. Under hypothesis $\left(H_{1}\right)$, the equilibrium states are

1. Pure liquid or gaseous states: $\alpha_{1}=0$ (resp. $\alpha_{2}=0$ ), with $\rho_{2}=\rho, \rho_{1}<\rho$ arbitrary (resp. $\rho_{1}=\rho, \rho<\rho_{2}$ arbitrary)

2. Coexistence states: $\alpha_{1} \alpha_{2} \neq 0$, with $\left(\rho_{1}, \rho_{2}\right)$ satisfying

$$
\mu\left(\rho_{1}\right)=\mu\left(\rho_{2}\right) \quad \text { and } \quad p\left(\rho_{1}\right)=p\left(\rho_{2}\right) .
$$

Proof. The case $\alpha_{1}=0$ corresponds to the saturation of the constraint $\alpha_{1}+\alpha_{2}=1$; see Equation (2.15). It leads to $\alpha_{2}=1$ and thus $\rho_{2}=\rho$ that is only the phase 2 is present. Conversely if $\alpha_{2}=1$ only the phase 1 remains.

On the other hand let us assume $\alpha_{1} \alpha_{2} \neq 0$. Then Equations (2.23) and (2.24) lead to the equality of the chemical potentials

$$
\mu\left(\rho_{1}\right)=\mu\left(\rho_{2}\right)=-\lambda_{\rho}
$$

Then the intensive Gibbs' relation (2.8) allows to rewrite the conditions (2.21) and (2.22) as

$$
\rho_{i} \mu\left(\rho_{i}\right)-p\left(\rho_{i}\right)+\lambda_{\alpha}+\lambda_{\rho} \rho_{i}=0, \quad i=1,2 .
$$

Since $-\lambda_{\rho}=\mu\left(\rho_{i}\right)$, this leads to the pressures equality

$$
p\left(\rho_{1}\right)=p\left(\rho_{2}\right)=\lambda_{\alpha} .
$$

To proceed further we need the following result for coexistence states.

Proposition 2.1. Under hypothesis $\left(H_{1}\right)$ and if $\alpha_{1} \alpha_{2} \neq 0$, the following propositions are equivalent and uniquely define the couple of densities $\rho_{1}^{*}<\rho_{2}^{*}$. 
1. The chemical potentials and the pressures are equal

$$
\begin{aligned}
& \mu\left(\rho_{1}^{*}\right)=\mu\left(\rho_{2}^{*}\right), \\
& p\left(\rho_{1}^{*}\right)=p\left(\rho_{2}^{*}\right) .
\end{aligned}
$$

2. The Maxwell's area rule on the chemical potential holds

$$
\int_{0}^{1} \mu\left(\rho_{2}+t\left(\rho_{1}-\rho_{2}\right)\right) d t=\mu\left(\rho_{1}^{*}\right)=\mu\left(\rho_{2}^{*}\right) .
$$

3. The difference of the Helmholtz free energies reads

$$
f\left(\rho_{2}^{*}\right)-f\left(\rho_{1}^{*}\right)=\mu\left(\rho_{1}^{*}\right)\left(\rho_{2}^{*}-\rho_{1}^{*}\right)=\mu\left(\rho_{2}^{*}\right)\left(\rho_{2}^{*}-\rho_{1}^{*}\right) .
$$

Proof. The identities (2.27) and (2.28) are equivalent since $f^{\prime}(\rho)=\mu(\rho)$; see Equation (2.9). Now assume Equations (2.25)-(2.26) hold. Then the intensive Gibbs' relation (2.8) gives Equation (2.28). Conversely, Equation (2.28) implies the chemical potentials equality and thus the pressures equality. Now the uniqueness of $\left(\rho_{1}^{*}, \rho_{2}^{*}\right)$ follows easily form a geometrical argument using the Maxwell's area rule.

The most famous characterizations of diphasic equilibria are Equations (2.25)-(2.26) and (2.27), although the latter is usually written in terms of pressure. We can recover this form by writing the intensive relations with the specific volume $\tau=V / M$ instead of $\rho$. The third relation (2.28) is not so classic but will be useful in the sequel.

The density $\rho_{1}^{*}$ (resp. $\rho_{2}^{*}$ ) separates the range of pure gaseous state and the range of metastable gas (resp. the range of pure liquid state and the range of metastable liquid), see Figure 2.1. These densities define the coexistence pressure $p^{*}$ and the coexistence chemical potential $\mu^{*}$ as follows:

$$
p^{*}=p\left(\rho_{1}^{*}\right)=p\left(\rho_{2}^{*}\right), \quad \mu^{*}=\mu\left(\rho_{1}^{*}\right)=\mu\left(\rho_{2}^{*}\right) .
$$

We emphasize that the necessary conditions for equilibrium contain both unstable (spinodal zone), metastable, and stable states. Nothing at this stage can make the difference, which turns out to be of dynamical nature, in a way we make precise now.

\section{Dynamical system and phase transition}

This section is devoted to the construction and the analysis of a dynamical system deduced from the optimality conditions given in Lemma 2.2 and Proposition 2.1. We will prove that the equilibria of this dynamical system are either pure liquid/vapor states, pure metastable states or coexistence states in the spinodal zone (that is states satisfying the properties (2.25)-(2.27) of Proposition 2.1). We emphasize that the difference between metastable states and coexistent states actually relies on the long-time dynamical behaviour of the solutions to the dynamical system. No static characterization can be given.

The section ends with numerical illustrations that assess the ability of the dynamical system to preserve metastable states.

3.1. Construction of the dynamical system. We want to construct a dynamical system which equilibria coincide with the equilibria of the optimization problem depicted in Lemma 2.2. To do so, we force the dynamical system to dissipate the Helmholtz free energy defined by

$$
\begin{aligned}
\mathcal{F}: \mathbb{R}^{+} \times \mathbb{R}^{+} \times \mathbb{R}^{+} & \rightarrow \mathbb{R} \\
\left(\rho, \rho_{1}, \rho_{2}\right) & \mapsto \alpha_{1}\left(\rho, \rho_{1}, \rho_{2}\right) f\left(\rho_{1}\right)+\alpha_{2}\left(\rho, \rho_{1}, \rho_{2}\right) f\left(\rho_{2}\right),
\end{aligned}
$$


under the optimization constraints (2.15) and (2.16).

Assuming that $\rho, \rho_{1}$, and $\rho_{2}$ are now time-dependent functions, we can compute the derivative of the total Helmholtz free-energy $\mathcal{F}$ with respect to time and deduce appropriate time derivatives of $\rho, \rho_{1}$, and $\rho_{2}$ such that $\mathcal{F}$ is dissipated in time. Moreover we want pure states (either liquid, vapor, or metastable) to be equilibria of the dynamical system. Hence we have forced the time derivatives of $\rho, \rho_{1}$, and $\rho_{2}$ to vanish in case of pure state (that is when $\alpha_{1} \alpha_{2}=0$ ).

For the sake of readability we denote

$$
\mathbf{r}=\left(\begin{array}{c}
\rho \\
\rho_{1} \\
\rho_{2}
\end{array}\right)
$$

the vector of admissible densities satisfying the assumption $\left(H_{1}\right)$. By the definition $(2.17)$ of the volume fractions $\alpha_{i}, i=1,2$, one has

$$
\nabla_{\mathbf{r}} \alpha_{1}(\mathbf{r})=-\nabla_{\mathbf{r}} \alpha_{2}(\mathbf{r})=\frac{1}{\rho_{1}-\rho_{2}}\left(\begin{array}{c}
1 \\
-\alpha_{1}(\mathbf{r}) \\
-\alpha_{2}(\mathbf{r})
\end{array}\right)
$$

From this and the definition (3.1) of $\mathcal{F}$, we easily get the gradient of the free-energy

$$
\nabla_{\mathbf{r}} \mathcal{F}(\mathbf{r})=\frac{1}{\rho_{1}-\rho_{2}}\left(\begin{array}{c}
f\left(\rho_{1}\right)-f\left(\rho_{2}\right) \\
\alpha_{1}(\mathbf{r})\left(\rho_{2}\left(\mu\left(\rho_{2}\right)-\mu\left(\rho_{1}\right)\right)+p\left(\rho_{1}\right)-p\left(\rho_{2}\right)\right) \\
\alpha_{2}(\mathbf{r})\left(\rho_{1}\left(\mu\left(\rho_{2}\right)-\mu\left(\rho_{1}\right)\right)+p\left(\rho_{1}\right)-p\left(\rho_{2}\right)\right)
\end{array}\right) .
$$

Note at once that it can be expressed in terms of relative free-energy

$$
\nabla_{\mathbf{r}} \mathcal{F}(\mathbf{r})=\frac{1}{\rho_{1}-\rho_{2}}\left(\begin{array}{c}
f\left(\rho_{1}\right)-f\left(\rho_{2}\right) \\
\alpha_{1}(\mathbf{r}) f\left(\rho_{2} \mid \rho_{1}\right) \\
-\alpha_{2}(\mathbf{r}) f\left(\rho_{1} \mid \rho_{2}\right)
\end{array}\right)
$$

where the relative free-energy of $\rho_{2}$ with respect to $\rho_{1}$ is defined by

$$
f\left(\rho_{2} \mid \rho_{1}\right):=f\left(\rho_{2}\right)-f\left(\rho_{1}\right)-\mu\left(\rho_{1}\right)\left(\rho_{2}-\rho_{1}\right) .
$$

We turn now to the definition of our dynamical system. Because of the mass conservation, we obviously impose that

$$
\dot{\rho}=0 \text {. }
$$

The main idea to proceed further is that we want the system to dissipate the total Helmholtz free-energy $\mathcal{F}$. Combining the definition (3.1) of $\mathcal{F}$ and the expression of $\nabla_{\mathbf{r}} \mathcal{F}(\mathbf{r})$, one computes the time derivative of $\mathcal{F}$ along some trajectory, that is

$$
\dot{\mathcal{F}}(\mathbf{r})=\frac{1}{\rho_{1}-\rho_{2}}\left(\alpha_{1}(\mathbf{r}) f\left(\rho_{2} \mid \rho_{1}\right) \dot{\rho}_{1}-\alpha_{2}(\mathbf{r}) f\left(\rho_{1} \mid \rho_{2}\right) \dot{\rho}_{2}\right)
$$

We now propose the following dynamical system

$$
\left\{\begin{array}{l}
\dot{\rho}=0 \\
\dot{\rho}_{1}=-\left(\rho-\rho_{1}\right)\left(\rho-\rho_{2}\right) f\left(\rho_{2} \mid \rho_{1}\right), \\
\dot{\rho}_{2}=+\left(\rho-\rho_{1}\right)\left(\rho-\rho_{2}\right) f\left(\rho_{1} \mid \rho_{2}\right) .
\end{array}\right.
$$


An easy computation shows that this system dissipates $\mathcal{F}$ along its trajectories (under the assumption $\left(H_{1}\right)$ ). Indeed using the expression (3.6) of $\dot{\mathcal{F}}$ and the equations of $\dot{\rho}_{1}$ and $\dot{\rho_{2}}$, one gets

$$
\dot{\mathcal{F}}(\mathbf{r})=-\left(\rho-\rho_{1}\right)\left[\alpha_{1}(\mathbf{r}) f\left(\rho_{2} \mid \rho_{1}\right)\right]^{2}+\left(\rho-\rho_{2}\right)\left[\alpha_{2}(\mathbf{r}) f\left(\rho_{1} \mid \rho_{2}\right)\right]^{2} .
$$

The multiplicative term $\left(\rho-\rho_{1}\right)\left(\rho-\rho_{2}\right)$ in the equations on $\dot{\rho}_{1}$ and $\dot{\rho}_{2}$ ensures that the right-hand side of the system vanishes in case of pure states (either pure liquid, vapor or metastable states).

REMARK 3.1. We emphasize that the choice of the right-hand side of Equation (3.7) is somewhat arbitrary. Other terms might be more efficient, but this one was chosen for its simplicity and its interpretation in terms of relative free-energy.

One can check easily that $\overbrace{\alpha_{1} \rho_{1}+\alpha_{2} \rho_{2}}=0$ so that it is consistent with the total mass conservation equation $\dot{\rho}=0$.

An equivalent dynamical system can be written in terms of the time derivatives of the volume fractions $\alpha_{i}$ and of the partial masses $\alpha_{i} \rho_{i}, i=1,2$. Accounting for the constraints (2.15) and (2.16), and for the system (3.7), some straightforward computations lead to

$$
\left\{\begin{aligned}
\dot{\alpha}_{1} & =-\dot{\alpha}_{2} \\
\overbrace{\alpha_{1} \rho_{1}} & =-\alpha_{1}^{2}\left(\rho-\rho_{1}\right) f\left(\rho_{2} \mid \rho_{1}\right)+\alpha_{2}^{2}\left(\rho-\rho_{2}\right) f\left(\rho_{1} \mid \rho_{2}\right), \\
& =\alpha_{1}^{2} \rho_{2}\left(\rho-\rho_{1}\right) f\left(\rho_{2} \mid \rho_{1}\right)+\alpha_{2}^{2} \rho_{1}\left(\rho-\rho_{2}\right) f\left(\rho_{1} \mid \rho_{2}\right) .
\end{aligned}\right.
$$

This formulation does not allow to compute single phase systems, since the determination of the partial densities $\rho_{i}$ is impossible when $\alpha_{i}=0$. Thus we rather use the dynamical system (3.7).

3.2. Equilibria of the dynamical system. The major result of this paragraph is that the attractors of the system are either pure liquid/vapor states, including metastable states, or the coexistence state defined by (2.26)-(2.27), see Proposition 2.1.

Proposition 3.1. The dynamical system (3.7) satisfies the following properties.

1. If the assumption $\left(H_{1}\right)$ is satisfied at $t=0$, then it is preserved in time (in particular, the assumption $\rho_{1}<\rho_{2}$ is preserved).

2. If $\alpha_{1}(0)=0$ (resp. $\left.\alpha_{1}(0)=1\right)$ then $\alpha_{1}(t)=0$ (resp. $\left.\alpha_{1}(t)=1\right)$, for all time.

Proof. First we address the preservation of the hypothesis $\left(H_{1}\right)$. Some straightforward computations lead to

$$
\dot{\rho}_{1}-\dot{\rho}_{2}=\left(\rho-\rho_{1}\right)\left(\rho-\rho_{2}\right)\left(\rho_{1}-\rho_{2}\right)\left(\mu\left(\rho_{2}\right)-\mu\left(\rho_{1}\right)\right)
$$

Thus if $\rho_{1}-\rho_{2}$ is zero at $t=0$ then this property is preserved for all time and the sign of the difference $\rho_{1}-\rho_{2}$ is also preserved. The property on the volume fraction $\alpha_{1}$ is proved in the same way noting that

$$
\dot{\alpha}_{1}(\mathbf{r})=-\alpha_{1}(\mathbf{r})\left(\rho-\rho_{1}\right)\left(\rho_{1}\left(\mu\left(\rho_{1}\right)-\mu\left(\rho_{2}\right)\right)-p\left(\rho_{1}\right)+p\left(\rho_{2}\right)\right) .
$$


We turn now to the characterization of the equilibria of the dynamical system. Since the total mass $\rho$ is constant in time, they are parametrized by $\rho$. Moreover it arises that their thermodynamic characterization can be given in terms of attraction basins. We refer the reader for graphical references to figures 3.1, 3.2, and 3.3, where attraction basins are drawn as hatched zones. The last two figures correspond to the gaseous phase, similar pictures can be drawn for the liquid phase.

Theorem 3.1. Assume that the initial data $\left(\rho(0), \rho_{1}(0), \rho_{2}(0)\right)$ of the system $(3.7)$ satisfy $\left(H_{1}\right)$. Then the equilibria are given by

1. spinodal zone: $\rho_{1}^{*}<\rho(0)<\rho_{2}^{*}$. The unique equilibrium is $\left(\rho_{1}^{*}, \rho_{2}^{*}\right)$ characterized by Proposition 2.1, with $\alpha_{i}$ given by Equation (2.17). The attraction basin is $(0, \rho) \times(\rho, 3)$.

2. pure gaseous states: $\rho(0)<\rho_{1}^{*}$ (resp. pure liquid states: $\rho(0)>\rho_{2}^{*}$ ). The set of equilibria is $\{\rho\} \times(\rho, 3)$, with $\alpha_{1}=1$ (resp. $(0, \rho) \times\{\rho\}$ with $\left.\alpha_{2}=1\right)$. The attraction basin is $(0, \rho) \times(\rho, 3)$.

3. metastable states: $\rho_{1}^{*} \leq \rho(0) \leq \rho^{-}$(resp. $\left.\rho^{+} \leq \rho(0) \leq \rho_{2}^{*}\right)$. There are two sets of equilibria

(a) perturbation within the phase: $\rho_{2}(0) \leq \rho^{-}\left(\right.$resp. $\left.\rho_{1}(0) \geq \rho^{+}\right)$. The set of equilibria is $\{\rho\} \times\left(\rho, \rho^{-}\right)$, with $\alpha_{1}=1$ (resp. $\left(\rho^{+}, \rho\right) \times\{\rho\}$ with $\left.\alpha_{2}=1\right)$. The attraction basin is then $(0, \rho) \times\left(\rho, \rho^{-}\right)$(resp. $\left.\left(\rho^{+}, \rho\right) \times(\rho, 3)\right)$.

(b) outside perturbation: $\rho^{-} \leq \rho_{2}(0) \leq \rho_{2}^{*}$ (resp. $\left.\rho^{+} \geq \rho_{1}(0) \geq \rho_{1}^{*}\right)$. There is a unique equilibrium $\left(\rho_{1}^{*}, \rho_{2}^{*}\right)$, characterized by Proposition 2.1, with $\alpha_{i}$ given by Equation (2.17). The attraction basin is $(0, \rho) \times\left(\rho^{-}, 3\right)$ (resp. $\left.\left(0, \rho^{+}\right) \times(\rho, 3)\right)$.

Proof. We look for Lyapunov functions for each case.

Spinodal zone. Let us define

$$
G(\mathbf{r})=\alpha_{1} f\left(\rho_{1}\right)+\alpha_{2} f\left(\rho_{2}\right)-\mu^{*}\left(\alpha_{1} \rho_{1}+\alpha_{2} \rho_{2}\right)+p^{*}\left(\alpha_{1}+\alpha_{2}\right),
$$

where $\mu^{*}$ and $p^{*}$ are defined by the Maxwell area rule (2.29) on the chemical potential $\mu$, and $\alpha_{i}$ are the functions of $\rho, \rho_{1}, \rho_{2}$ given by Equation (2.17). The intensive version of the Gibbs' relation (2.8) implies that $G\left(\mathbf{r}^{*}\right)=0$ where $\mathbf{r}^{*}=\left(\rho, \rho_{1}^{*}, \rho_{2}^{*}\right)^{T}$. Straightforward computations show that $\nabla_{\mathbf{r}} G=\nabla_{\mathbf{r}} \mathcal{F}-\left(\mu^{*}, 0,0\right)^{T}$, this implies that $\nabla_{\mathbf{r}} G\left(\mathbf{r}^{*}\right)=0$, and that $\dot{G}(\mathbf{r}(t)) \leq 0$ since the free-energy is dissipated. Hence the function $G$ is a Lyapunov function on the admissible domain defined by $\left(H_{1}\right)$, which contains the unique equilibrium $\mathbf{r}^{*}$, see Figure 3.1.

Pure stable states. The expected equilibrium states are now $\overline{\mathbf{r}}=\left(\rho, \rho, \rho_{2}\right)$ for any $\rho_{2} \geq \rho$, see Figure 3.2. We introduce the function

$$
G^{(1)}(\mathbf{r})=f\left(\rho_{1}\right)-\bar{\mu} \rho_{1}+\bar{p}, \quad \text { where } \quad \bar{\mu}=\mu(\rho), \bar{p}=p(\rho) .
$$

Once again, the Gibbs' relation leads to $G^{(1)}(\overline{\mathbf{r}})=0$ for any equilibrium $\overline{\mathbf{r}}$, and we easily get $\nabla_{\mathbf{r}} G^{(1)}(\overline{\mathbf{r}})=0$. Now we have

$$
\frac{d}{d t} G^{(1)}(\mathbf{r}(t))=-\left(\mu\left(\rho_{1}\right)-\mu(\rho)\right)\left(\rho-\rho_{1}\right)\left(\rho-\rho_{2}\right) f\left(\rho_{2} \mid \rho_{1}\right)
$$

Since $\rho_{1} \leq \rho \leq \rho_{2}$ and $\mu$ is nondecreasing on $\left.] 0, \rho_{1}^{*}\right]$ the right-hand side in the preceding relation is nonpositive if $f\left(\rho_{2} \mid \rho_{1}\right) \geq 0$. But using again the Gibbs' relation and Equation (3.5), this can be rewritten

$$
f\left(\rho_{2}\right)-f\left(\rho_{1}\right) \geq \mu\left(\rho_{1}\right)\left(\rho_{2}-\rho_{1}\right) .
$$




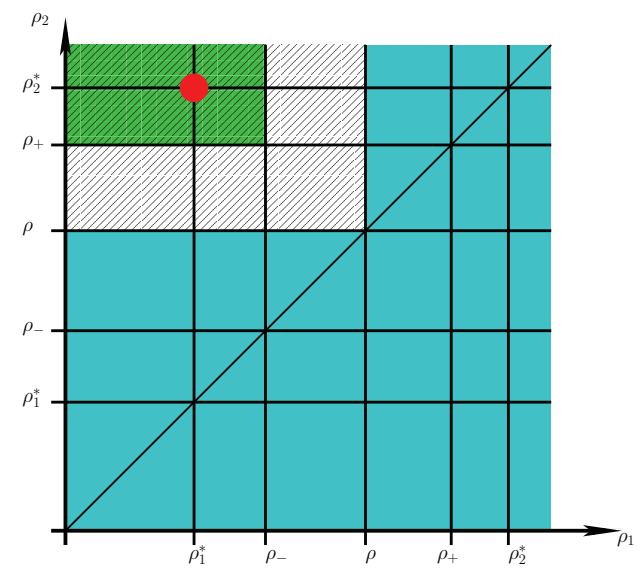

FIG. 3.1. Spinodal states. The blue area refers to unattainable states according to the hypothesis $\left(H_{1}\right)$. The attraction basin is the hatched area $(/ / /)$. The unique attraction point $\left(\rho_{1}^{*}, \rho_{2}^{*}\right)$ appears in red. The green zone corresponds to the invariant hyperbolicity region (see Section 4.4).

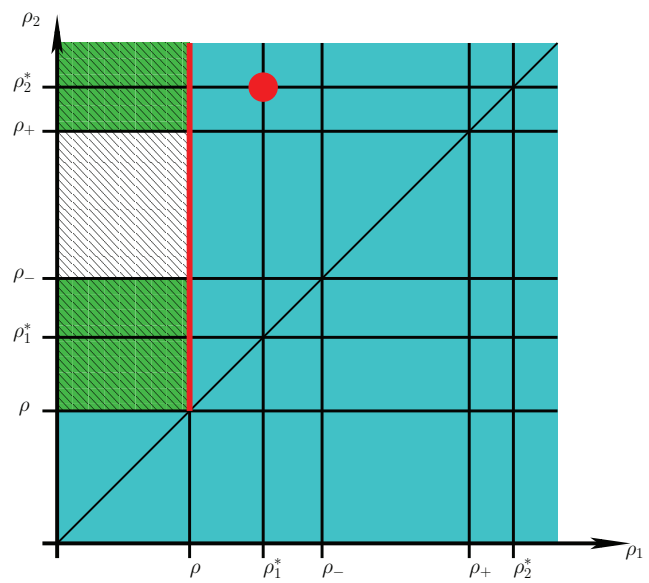

FIG. 3.2. Pure gaseous states. The blue area refers to unattainable states according to $\left(H_{1}\right)$. The attraction basin is the hatched area $\backslash \backslash \backslash)$. The set of attraction points appears in red. The two green zones correspond to the invariant hyperbolicity regions (see Section 4.4).

This convexity inequality holds true for all $\left(\rho_{1}, \rho_{2}\right)$ such that $\rho_{1} \leq \rho_{1}^{*}$, by the very definition of $\rho_{1}^{*}$ (see the definition $(2.20)$ of $F^{* *}$ ). Since we consider pure liquid states, one has $\rho_{1} \leq \rho \leq \rho_{1}^{*}$. Hence $G^{(1)}$ is dissipated in time, leading to the conclusion.

Metastable states. Two types of equilibria are encountered in this situation, with two distinct attraction basins, see Figure 3.3.

- the metastable basin, which appears with $\backslash \backslash \backslash$ hatches in the figure, corresponds to perturbation of a given state within the same phase. It is actually an attraction basin, because the function $G^{(1)}$ defined above in Equation (3.12) is also a Lyapunov function in this domain. Indeed the convexity argument still holds true for any $\left(\rho_{1}, \rho_{2}\right)$ such that $\rho_{2} \leq \rho^{-}$, since $f$ is convex below $\rho^{-}$.

- the unstable basin, corresponding to perturbations of the state by the other 


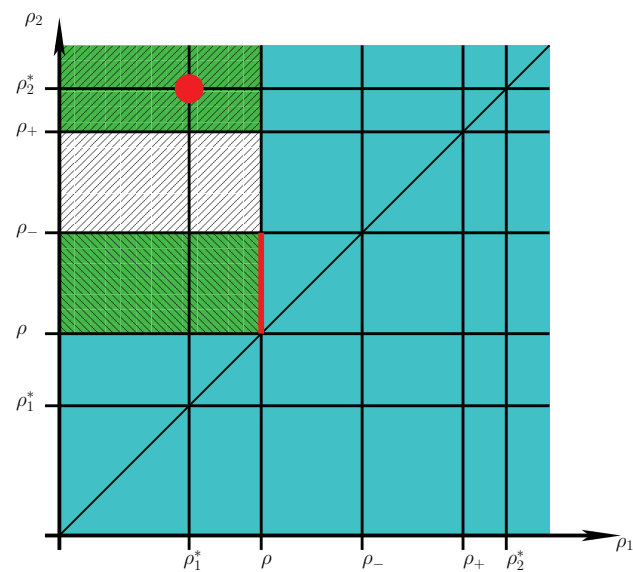

FIG. 3.3. Pure gaseous metastable states. The blue area refers to unattainable states according to $\left(H_{1}\right)$. The attraction points appear in red. The corresponding attraction basins are the hatched areas. For any state belonging to the hatched area $\bigwedge \backslash \backslash$ ), the attraction points are metastable vapor sates such that $\left\{\rho_{1}=\rho\right\}$. For any state belonging to the hatched area $(/ / /)$, the attraction point is the coexistence state $\left(\rho_{1}^{*}, \rho_{2}^{*}\right)$. The two green zones correspond to the invariant hyperbolicity regions (see Section 4.4).

phase (/// hatched zone). This basin is governed by the same Lyapunov function as for the spinodal zone (3.11).

REMARK 3.2. This is a formalization of the remark in Landau \& Lifshitz $[25, \S 21]$ "[... wwe must distinguish between metastable and stable equilibrium states. A body in a metastable state may not return to it after a sufficient deviation" .

\begin{tabular}{|l|c|c|}
\hline$\rho$ & $p(\rho)$ & $\mu(\rho)$ \\
\hline$\rho^{-}=0.5810799446067$ & 0.62055388470356498 & -3.68447967881140137 \\
\hline$\rho^{+}=1.4888047089018$ & 0.04962960899844759 & -4.24339302065563029 \\
\cline { 1 - 1 }$\rho_{1}^{*}=0.31972996451885$ & 0.504491649787487 & -3.97717851100986 \\
\cline { 1 - 1 }$\rho_{2}^{*}=1.8071403273364$ & & \\
\hline
\end{tabular}

TABLE 3.1. Characteristic densities at $T=0.85$ and corresponding pressure and chemical potential values

\subsection{Numerical illustrations of the dynamical system behavior}

We provide in this paragraph some numerical examples to illustrate the behavior of the dynamical system (3.7). First we give the characteristic values of the reduced van der Waals EoS at a fixed nondimensionalized temperature $T=0.85$. Figure 3.4 presents the corresponding isothermal curve in the $(\rho, \mu)$ plane.

The densities $\rho^{-}$and $\rho^{+}$correspond to the extrema of the chemical potential. The densities $\rho_{1}^{*}$ and $\rho_{2}^{*}$ are obtained by the Maxwell's equal area rule construction on the chemical potential (2.27) or equivalently by solving the system (2.25)-(2.26).

Table 3.1 contains the values of the characteristic densities and the corresponding values of the pressure and chemical potential at $T=0.85$.

Using a backward differentiation formula (provided by Scilab for stiff problem), we provide numerical illustrations of the attraction basins depicted in Theorem 3.1. To do so, we fix 50 initial values of $\rho(0)$ in a given interval and set the partial densities $\rho_{i}(0)$ 


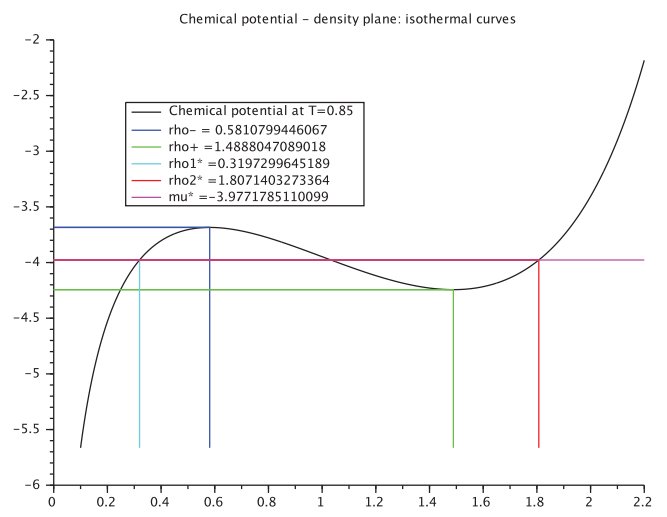

FIG. 3.4. Isotherm curve in the $(\rho, \mu)$ plane at $T=0.85$.
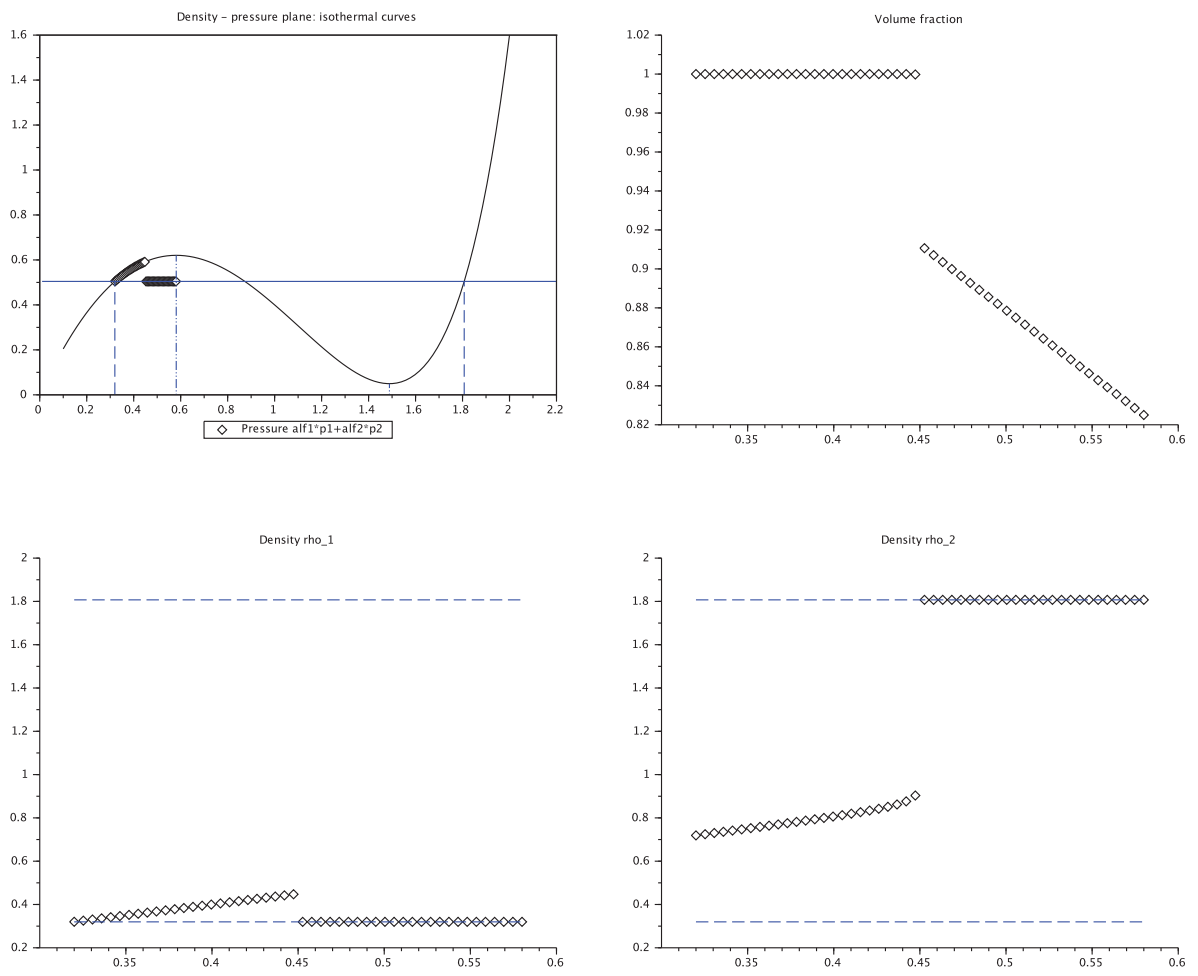

FIG. 3.5. Numerical illustration of a perturbation within the metastable vapor zone. The initial density $\rho(0)$ takes on 50 values in $\left[\rho_{1}^{*}, \rho^{-}\right]$and $\rho_{i}(0)$ are perturbations of $\rho(0)$ under the assumption $\left(H_{1}\right)$. The top-left figure corresponds to the mixture pressure at final time $T_{f}=10^{3}$. For densities $\rho(0)<0.45$, the pressure coincides with the reduced van der Waals pressure, while for $\rho(0)>0.45$ it matches with the Maxwell line. One notices that the volume fraction $\alpha_{1}\left(T_{f}\right)$ is either constant equal to 1 (for $\rho(0)<0.45)$, correspondong to pure phase 1, or takes on values in ]0,1[, which means that the system reached the coexistent state. 
as perturbations of $\rho(0)$. Then the dynamical system is solved for a final time $T_{f}=10^{3}$ with a time step set to $10^{-3} \mathrm{~s}$. We provide the graphs of the quantities $\alpha_{1}\left(T_{f}\right), \rho_{i}\left(T_{f}\right)$ and $\alpha_{1}\left(T_{f}\right) p\left(\rho_{1}\left(T_{f}\right)\right)+\alpha_{2}\left(T_{f}\right) p\left(\rho_{2}\left(T_{f}\right)\right)$, plotted with respect to the density $\rho(0)$, for each one of the 50 initial conditions $\left(\rho(0), \rho_{1}(0), \rho_{2}(0)\right)$. The mixture pressure profile is compared with the classical van der Waals pressure curve and the Maxwell line.

Metastable states: The initial density $\rho(0)$ takes on 50 values in $\left[\rho_{1}^{*}, \rho^{-}\right]$. For this computation, we set $\rho_{1}(0)=\rho(0)-0.1$ and $\rho_{2}(0)=\rho(0)+0.2$ so that we can observe the perturbation within and outside the metastable vapor zone. According to Figure 3.5 (top-left), the mixture pressure presents two different parts: the left part (for $\rho<0.45$ ) remains on the van der Waals pressure curve and a second part coincides with the Maxwell line. The first part corresponds to the perturbation of the metastable vapor state within the phase, while the right part corresponds to the perturbation outside the metastable vapor, that is when $\rho^{-} \leq \rho_{2}(0) \leq \rho_{2}^{*}$. One can check on the volume fraction curve (see Figure 3.5(top-right)) that $\alpha_{1}\left(T_{f}\right)=1$ for $\rho(0) \leq 0.45$, that is only the phase 1 is present. Then $0<\alpha_{1}<1$ and the corresponding final partial densities $\rho_{i}\left(T_{f}\right), i=1,2$, coincide with the densities $\rho_{1}^{*}$ and $\rho_{2}^{*}$ respectively, which explains that the pressure matches with the Maxwell line.
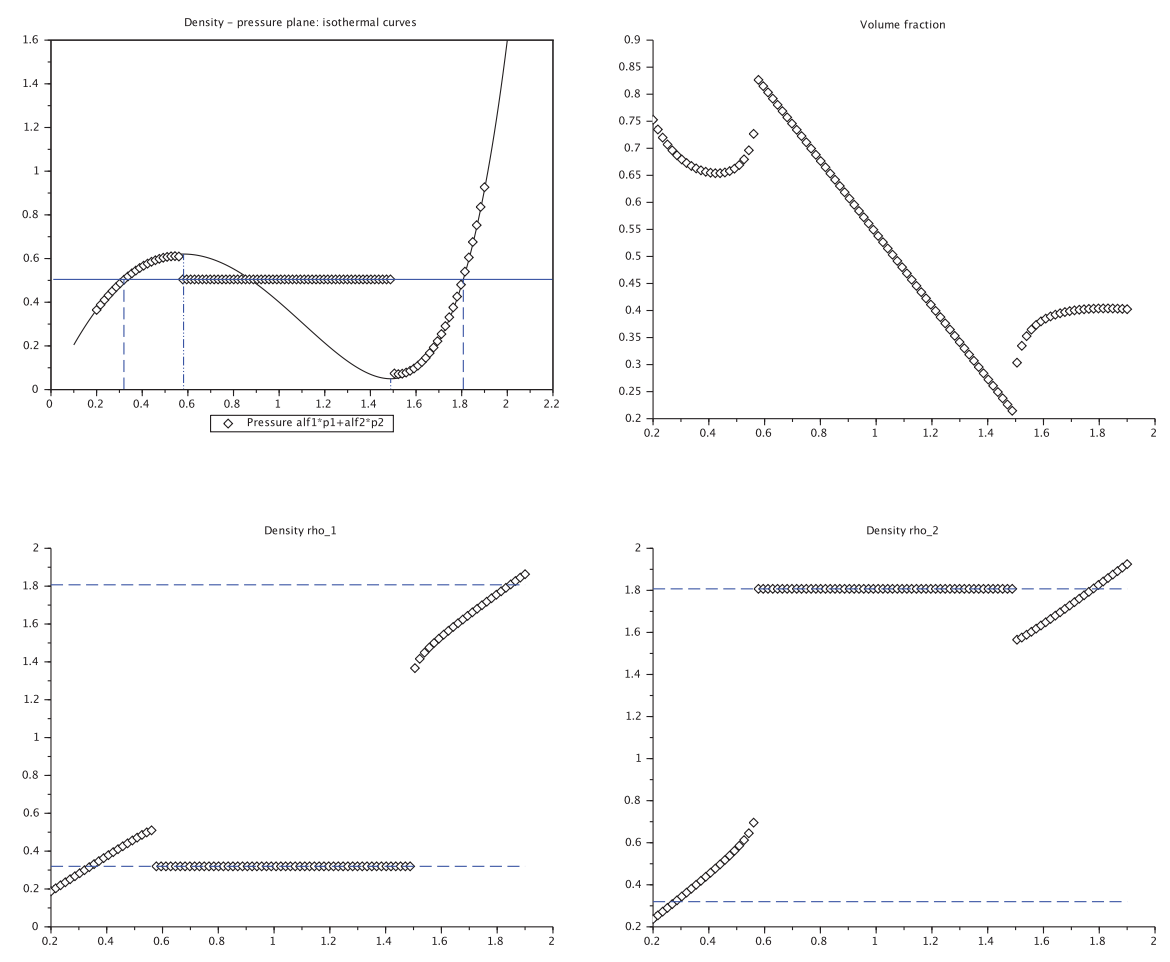

FIG. 3.6. Numerical illustration of a perturbation of $\rho(0)$ in the whole domain. The mixture pressure $\alpha_{1}\left(T_{f}\right) p\left(\rho_{1}\left(T_{f}\right)\right)+\alpha_{2}\left(T_{f}\right) p\left(\rho_{2}\left(T_{f}\right)\right)$ coincides with the admissible branches of the reduced van der Waals pressure in the pure liquid/vapor states, including metastable state. In the spinodal zone it corresponds to the Maxwell line.

Perturbation of the density $\rho$ in the whole domain: Figure 3.6 corresponds to an initial density $\rho(0)$ which takes on 50 values between 0.2 and 1.8 , while $\rho_{1}(0)=$ 
$\rho(0)-0.1$ and $\rho_{2}(0)=\rho(0)+0.1$. One observes that an initial perturbation of the density $\rho(0)$ leads to final states which belong to either pure vapor/liquid states, including metastable states, or the coexistent state. Hence the mixture pressure coincides with the admissible branches of the van der Waals pressure curve or with the Maxwell line.

However the convergence is not obvious for $\rho(0)$ close to $\rho^{-}$on the left (resp. to $\rho^{+}$on the right). This can be observed in Figure 3.6(top-right) (plot of the volume fraction), where the parts on the left and on the right should be straight lines. Actually, since for $\rho$ close to $\rho^{-}$the perturbation chosen is in the spinodal zone, we expect the equilibrium to be on the Maxwell line, which is not the case. We suppose that the final time is not large enough to ensure the actual convergence. As a matter of a fact, we observed that the requested time to reach convergence is larger in the metastable zone than in the spinodal one.

\section{The isothermal model}

This section is devoted to the definition and study of a $4 \times 4$ van der Waals isothermal two-phase flow model. Since we are interested in the modeling of phase transitions with possible metastable states, the liquid-vapor flows that we consider are submitted to strong thermodynamic perturbations. Hence we propose to depict the dynamic of the flows by a compressible averaged model, namely Euler type equations.

In order to model phase transitions, the hydrodynamic part of the model is classically coupled with a relaxation source term which carries on the mass transfer. Since we wish to take into account possible metastable states, the equilibria of the source term have to be either pure liquid/vapor states, metastable states, or the coexistence state given by Equations (2.26)-(2.27). Hence we propose a coupling between the dynamical system (3.7) introduced in the previous section and a modified version of the isothermal two-phase model proposed in [3].

After defining the model, we study several properties of the system, such as existence of a decreasing energy, hyperbolicity, and Riemann invariants for the homogeneous system. Notice at once that we have only partial results for hyperbolicity, as noticed before in the literature, because of the spinodal zone. This leads to a formal study of invariant hyperbolicity domains in the last subsection.

4.1. Definition of the model. The basic isothermal Euler system contains the balance equations accounting for the conservation of the total mass and the total momentum of the two-phase flow. We propose to extend this system with two equations describing the evolution of the partial densities $\rho_{1}$ and $\rho_{2}$ which are now functions of time $t$ and space $x$. The two phases evolve with the same velocity $u$. The momentum equation involves a pressure term which is the mixture pressure $\alpha_{1} p\left(\rho_{1}\right)+\alpha_{2} p\left(\rho_{2}\right)$. Here $\alpha_{1}$ and $\alpha_{2}$ are given by Equation (2.17) but for the sake of readability we skip this dependence in what follows.

The system we propose is the following

$$
\left\{\begin{array}{l}
\partial_{t} \rho+\partial_{x}(\rho u)=0 \\
\partial_{t} \rho_{1}+\partial_{x}\left(\rho_{1} u\right)=-\frac{1}{\varepsilon}\left(\rho-\rho_{1}\right)\left(\rho-\rho_{2}\right) f\left(\rho_{2} \mid \rho_{1}\right), \\
\partial_{t} \rho_{2}+\partial_{x}\left(\rho_{2} u\right)=\frac{1}{\varepsilon}\left(\rho-\rho_{1}\right)\left(\rho-\rho_{2}\right) f\left(\rho_{1} \mid \rho_{2}\right), \\
\partial_{t}(\rho u)+\partial_{x}\left(\rho u^{2}+\alpha_{1} p\left(\rho_{1}\right)+\alpha_{2} p\left(\rho_{2}\right)\right)=0,
\end{array}\right.
$$

where $\varepsilon$ is a relaxation parameter that determines the rate at which the chemical potentials and pressures of the two phases reach equilibrium. The chemical potential $\mu$ and the pressure $p$ follow the van der Waals model (2.11). The source terms on the 
partial densities equations are exactly those of the system (3.7), and involve the relative free-energy $f\left(\rho_{i} \mid \rho_{j}\right)$ which is defined in Equation (3.5). System (4.1) is supplemented with initial conditions on the velocity $u$ and on the densities $\rho$ and $\rho_{i}, i=1,2$ satisfying the assumption $\left(H_{1}\right)$.

Combining the mass conservation Equation $(4.1)_{1}$ and the equations on the partial densities $\rho_{i}, i=1,2$, one can compute the equation satisfied by the volume fraction $\alpha_{1}$

$$
\partial_{t} \alpha_{1}+u \partial_{x} \alpha_{1}=\frac{1}{\varepsilon} \frac{\left(\rho-\rho_{1}\right)\left(\rho-\rho_{2}\right)}{\rho_{1}-\rho_{2}}\left[\alpha_{1} f\left(\rho_{2} \mid \rho_{1}\right)-\alpha_{2} f\left(\rho_{1} \mid \rho_{2}\right)\right]
$$

From the system (4.1) one can also recover the two equations on the partial masses $\alpha_{i} \rho_{i}$ and deal with a system of the classical form

$$
\left\{\begin{array}{l}
\partial_{t} \rho+\partial_{x}(\rho u)=0 \\
\partial_{t}\left(\alpha_{1} \rho_{1}\right)+\partial_{x}\left(\alpha_{1} \rho_{1} u=\frac{1}{\varepsilon} \frac{\left(\rho-\rho_{1}\right)\left(\rho-\rho_{2}\right)}{\rho_{1}-\rho_{2}}\left[\alpha_{1} \rho_{2} f\left(\rho_{2} \mid \rho_{1}\right)-\alpha_{2} \rho_{1} f\left(\rho_{1} \mid \rho_{2}\right)\right]\right. \\
\partial_{t}\left(\alpha_{2} \rho_{2}\right)+\partial_{x}\left(\alpha_{2} \rho_{2} u=-\frac{1}{\varepsilon} \frac{\left(\rho-\rho_{1}\right)\left(\rho-\rho_{2}\right)}{\rho_{1}-\rho_{2}}\left[\alpha_{1} \rho_{2} f\left(\rho_{2} \mid \rho_{1}\right)-\alpha_{2} \rho_{1} f\left(\rho_{1} \mid \rho_{2}\right)\right]\right. \\
\partial_{t}(\rho u)+\partial_{x}\left(\rho u^{2}+\alpha_{1} p\left(\rho_{1}\right)+\alpha_{2} p\left(\rho_{2}\right)\right)=0 .
\end{array}\right.
$$

In the present paper we choose to focus on the system (4.1), which allows us to define the densities $\rho_{i}$ even when $\alpha_{i}=0$, which is not the case in the last system.

REMARK 4.1. An interesting feature is that the system boils down to the classical $p$-system in pure phases that is when $\alpha_{1} \alpha_{2}=0$, including the metastable regions.

4.2. Hyperbolicity and entropy for the homogeneous system. We introduce the mechanical energy

$$
\mathcal{E}\left(\rho, \rho_{1}, \rho_{2}, u\right)=\frac{\rho u^{2}}{2}+\mathcal{F}\left(\rho, \rho_{1}, \rho_{2}\right)
$$

where the total Helmholtz free-energy $\mathcal{F}$ is defined by Equation (3.1). The first result we obtain is the decrease in time of this energy.

Proposition 4.1. The function $\mathcal{E}$, defined in Equation (4.3), satisfies the following equation

$$
\partial_{t} \mathcal{E}+\partial_{x}\left(u\left(\mathcal{E}+\alpha_{1} p\left(\rho_{1}\right)+\alpha_{2} p\left(\rho_{2}\right)\right) \leq 0\right.
$$

Proof. On the one hand, using the notation (3.2), one has

$$
\partial_{t} \mathcal{F}(\mathbf{r})=\nabla_{\mathbf{r}} \mathcal{F}(\mathbf{r}) \partial_{t} \mathbf{r}=-\nabla_{\mathbf{r}} \mathcal{F}(\mathbf{r}) \partial_{x}(u \mathbf{r})+\nabla_{\mathbf{r}} \mathcal{F}(\mathbf{r}) \cdot Q
$$

where $Q=\frac{1}{\varepsilon}\left(0,-\left(\rho-\rho_{1}\right)\left(\rho-\rho_{2}\right) f\left(\rho_{2} \mid \rho_{1}\right),\left(\rho-\rho_{1}\right)\left(\rho-\rho_{2}\right) f\left(\rho_{1} \mid \rho_{2}\right)\right)^{t}$. Hence it comes

$$
\partial_{t} \mathcal{F}(\mathbf{r})=-u \partial_{x} \mathcal{F}(\mathbf{r})-\nabla_{\mathbf{r}} \mathcal{F}(\mathbf{r}) \cdot \mathbf{r} \partial_{x} u+\nabla_{\mathbf{r}} \mathcal{F}(\mathbf{r}) \cdot Q
$$

Now the expression $\nabla_{\mathbf{r}} \mathcal{F}(\mathbf{r})$ given in Equation (3.4) leads to

$$
\nabla_{\mathbf{r}} \mathcal{F}(\mathbf{r}) \cdot \mathbf{r}=\frac{1}{\rho_{1}-\rho_{2}}\left(\left(\left(f\left(\rho_{1}\right)-f\left(\rho_{2}\right)\right) \rho+\alpha_{1} \rho_{1} f\left(\rho_{2} \mid \rho_{1}\right)-\alpha_{2} \rho_{2} f\left(\rho_{1} \mid \rho_{2}\right)\right) .\right.
$$


Because of the definition of the relative free-energy (3.5), this yields

$$
\nabla_{\mathbf{r}} \mathcal{F}(\mathbf{r}) \cdot \mathbf{r}=\mathcal{F}(\mathbf{r})+\alpha_{1} p\left(\rho_{1}\right)+\alpha_{2} p\left(\rho_{2}\right) .
$$

Thus one has

$$
\begin{aligned}
\partial_{t} \mathcal{F}(\mathbf{r}) & =-u \partial_{x} \mathcal{F}(\mathbf{r})-\left(\mathcal{F}(\mathbf{r})+\alpha_{1} p\left(\rho_{1}\right)+\alpha_{2} p\left(\rho_{2}\right)\right) \partial_{x} u+\nabla_{\mathbf{r}} \mathcal{F}(\mathbf{r}) \cdot Q \\
& =-\partial_{x}(\mathcal{F}(\mathbf{r}) u)-\left(\alpha_{1} p\left(\rho_{1}\right)+\alpha_{2} p\left(\rho_{2}\right)\right) \partial_{x} u+\nabla_{\mathbf{r}} \mathcal{F}(\mathbf{r}) \cdot Q
\end{aligned}
$$

On the other hand, a classical Euler type computation gets

$$
\partial_{t} \frac{\rho u^{2}}{2}+\partial_{x}\left(u \frac{\rho u^{2}}{2}\right)+u \partial_{x}\left(\alpha_{1} p\left(\rho_{1}\right)+\alpha_{2} p\left(\rho_{2}\right)\right)=0 .
$$

Combining the previous two relations gives

$$
\partial_{t} \mathcal{E}+\partial_{x}\left(u\left(\mathcal{E}+\alpha_{1} p\left(\rho_{1}\right)+\alpha_{2} p\left(\rho_{2}\right)\right)\right)=\mathcal{F}(\mathbf{r}) \cdot Q \leq 0,
$$

where the final inequality follows again from the expression (3.4) of $\nabla_{\mathbf{r}} \mathcal{F}(\mathbf{r})$.

However, the function $\mathcal{E}$ is not convex everywhere, so that it cannot be considered as a mathematical entropy. Indeed $\mathcal{E}$ is convex where $\mathcal{F}$ is, and we have the following result.

THEOREM 4.1. The total Helmholtz free-energy $\mathcal{F}$ defined by Equation (3.1) is convex for $\rho \in] 0,3[, u \in \mathbb{R}$ and

- $\left(\rho_{1}, \rho_{2}\right) \in(0, \rho) \times\left(\left(\rho, \rho^{-}\right) \cup\left(\rho^{+}, 3\right)\right)$, if $\rho \leq \rho^{-}$

- $\left(\rho_{1}, \rho_{2}\right) \in\left(0, \rho^{-}\right) \times\left(\rho^{+}, 3\right)$, if $\rho \in\left(\rho^{-}, \rho^{+}\right)$

- $\left(\rho_{1}, \rho_{2}\right) \in\left(\left(0, \rho^{-}\right) \times\left(\rho^{+}, \rho\right)\right) \times(\rho, 3)$, if $\rho \geq \rho^{+}$

Proof. The function $\mathcal{F}$ is a convex combination of $f\left(\rho_{1}\right)$ and $f\left(\rho_{2}\right)$ where $f$ is the intensive Helmholtz free energy (2.8). By definition of $\rho_{-}$and $\rho_{+}$(see Figure 2.1), $f$ is convex on $\left(0, \rho^{-}\right] \cup\left[\rho^{+}, 3\right)$, so the result follows.

We turn now to the determination of the eigenvalues of the homogeneous system (4.1). If we set $\mathbf{Y}=\left(\rho, \rho_{1}, \rho_{2}, u\right)$, for smooth solutions, the homogeneous system can be written as

$$
\partial_{t} \mathbf{Y}+A(\mathbf{Y}) \partial_{X} \mathbf{Y}=0
$$

where the matrix $A(\mathbf{Y})$ is defined by

$$
A(\mathbf{Y})=\left(\begin{array}{cccc}
u & 0 & 0 & \rho \\
0 & u & 0 & \rho_{1} \\
0 & 0 & u & \rho_{2} \\
A_{1}(\mathbf{Y}) & A_{2}(\mathbf{Y}) & A_{3}(\mathbf{Y}) & u
\end{array}\right)
$$

where

$$
\begin{aligned}
& A_{1}(\mathbf{Y})=\frac{p\left(\rho_{1}\right)-p\left(\rho_{2}\right)}{\rho\left(\rho_{1}-\rho_{2}\right)} \\
& A_{2}(\mathbf{Y})=\frac{\alpha_{1}}{\rho\left(\rho_{1}-\rho_{2}\right)}\left(p\left(\rho_{2}\right)-p\left(\rho_{1}\right)\right)+\frac{\alpha_{1}}{\rho} p^{\prime}\left(\rho_{1}\right), \\
& A_{3}(\mathbf{Y})=\frac{\alpha_{2}}{\rho\left(\rho_{1}-\rho_{2}\right)}\left(p\left(\rho_{2}\right)-p\left(\rho_{1}\right)\right)+\frac{\alpha_{2}}{\rho} p^{\prime}\left(\rho_{2}\right) .
\end{aligned}
$$


The characteristic equation of $A(\mathbf{Y})$ is given by

$$
(u-\lambda)^{2}(u-c-\lambda)(u+c-\lambda),
$$

with the speed of sound

$$
c:=c(\mathbf{r})=\sqrt{\frac{1}{\rho}\left(\alpha_{1}\left(\rho, \rho_{1}, \rho_{2}\right) \rho_{1} p^{\prime}\left(\rho_{1}\right)+\alpha_{2}\left(\rho, \rho_{1}, \rho_{2}\right) \rho_{2} p^{\prime}\left(\rho_{2}\right)\right)} .
$$

Thus we obtain three distinct eigenvalues for the matrix $A(\mathbf{Y})$ :

$$
\lambda_{1}(\mathbf{Y})=u-c, \quad \lambda_{2}(\mathbf{Y})=\lambda_{3}(\mathbf{Y})=u, \quad \lambda_{4}(\mathbf{Y})=u+c .
$$

Note that the eigenvalues are real if $\mathbf{r}$ satisfies

$$
\alpha_{1}(\mathbf{r}) \rho_{1} p^{\prime}\left(\rho_{1}\right)+\alpha_{2}(\mathbf{r}) \rho_{2} p^{\prime}\left(\rho_{2}\right) \geq 0 .
$$

Accounting on relations (2.9), it is equivalent to the following hyperbolicity condition

$$
\alpha_{1}(\mathbf{r}) \rho_{1}^{2} \mu^{\prime}\left(\rho_{1}\right)+\alpha_{2}(\mathbf{r}) \rho_{2}^{2} \mu^{\prime}\left(\rho_{2}\right) \geq 0 .
$$

The right eigenvectors $r_{i}(\mathbf{Y}), i=1, \ldots, 4$, that satisfy $A(\mathbf{Y}) r_{i}(\mathbf{Y})=\lambda_{i}(\mathbf{Y}) r_{i}(\mathbf{Y})$ can be chosen as

$$
r_{1}(\mathbf{Y})=\left(\begin{array}{c}
-\frac{\rho}{c} \\
-\frac{\rho_{1}}{c} \\
-\frac{\rho_{2}}{c} \\
1
\end{array}\right), \quad r_{2}(\mathbf{Y})=\left(\begin{array}{c}
-\frac{A_{3}}{A_{1}} \\
0 \\
1 \\
0
\end{array}\right), \quad r_{3}(\mathbf{Y})=\left(\begin{array}{c}
-\frac{A_{2}}{A_{1}} \\
1 \\
0 \\
0
\end{array}\right), \quad r_{4}(\mathbf{Y})=\left(\begin{array}{c}
\frac{\rho}{c} \\
\frac{\rho_{1}}{c} \\
\frac{\rho_{2}}{c} \\
1
\end{array}\right),
$$

where the quantities $A_{1}, A_{2}$ and $A_{3}$ are defined in Equation (4.7).

If the densities $\rho, \rho_{1}$, and $\rho_{2}$ satisfy Equations $\left(H_{1}\right)$ and $(4.10)$, the matrix $A(\mathbf{Y})$ is diagonalizable in $\mathbb{R}$ and its eigenvectors span the whole space $\mathbb{R}^{4}$ so that the system is hyperbolic.

4.3. Structure of the waves. In this paragraph we study the structure of the waves. Assuming that the densities $\rho, \rho_{1}$, and $\rho_{2}$ satisfy Equations $\left(H_{1}\right)$ and (4.10), one can observe that the waves are either genuinely nonlinear or linearly degenerate.

Straightforward computations lead to the following property which will be useful in the sequel.

Proposition 4.2. The speed of sound c, function of state $\mathbf{r}$, satisfies the following properties relations

$$
\begin{aligned}
& \nabla p(\mathbf{r}) \cdot \mathbf{r}=\rho c^{2}(\mathbf{r}), \\
& \nabla c(\mathbf{r})=\frac{1}{2 c(\mathbf{r})}\left(\begin{array}{c}
-\frac{c(\mathbf{r})^{2}}{\rho}+\frac{1}{\rho\left(\rho_{1}-\rho_{2}\right)}\left(\rho_{1} p^{\prime}\left(\rho_{1}\right)-\rho_{2} p^{\prime}\left(\rho_{2}\right)\right) \\
\frac{\alpha_{1}(\mathbf{r})}{\rho}\left(\frac{\rho_{2} p^{\prime}\left(\rho_{2}\right)-\rho_{1} p^{\prime}\left(\rho_{1}\right)}{\rho_{1}-\rho_{2}}+p^{\prime}\left(\rho_{1}\right)+\rho_{1} p^{\prime \prime}\left(\rho_{1}\right)\right) \\
\frac{\alpha_{2}(\mathbf{r})}{\rho}\left(\frac{\rho_{2} p^{\prime}\left(\rho_{2}\right)-\rho_{1} p^{\prime}\left(\rho_{1}\right)}{\rho_{1}-\rho_{2}}+p^{\prime}\left(\rho_{2}\right)+\rho_{2} p^{\prime \prime}\left(\rho_{2}\right)\right)
\end{array}\right) .
\end{aligned}
$$


Let us start with the waves associated to the wave speed $u-c$ and $u+c$.

Proposition 4.3. The characteristic fields associated to the waves speed $\lambda_{1}(\mathbf{Y})=$ $u-c$ and $\lambda_{4}(\mathbf{Y})=u+c$ are genuinely nonlinear i.e. $\nabla_{\mathbf{Y}} \lambda_{1}(\mathbf{Y}) \cdot r_{1}(\mathbf{Y}) \neq 0$ and $\nabla_{\mathbf{Y}} \lambda_{4}(\mathbf{Y})$. $r_{4}(\mathbf{Y}) \neq 0$ for admissible state vector $\mathbf{Y}$ that is for densities $\left(\rho, \rho_{1}, \rho_{2}\right)$ satisfying $\left(H_{1}\right)$ and (4.10).

Proof. We introduce the notation $D(\mathbf{r})=(c(\mathbf{r}))^{2}$. We consider the wave associated to the eigenvalue $\lambda_{1}(\mathbf{Y})$. One has

$$
\begin{aligned}
\nabla_{\mathbf{Y}} \lambda_{1}(\mathbf{Y}) \cdot r_{1}(\mathbf{Y}) & =\left(\begin{array}{c}
\nabla_{\mathbf{r}} c(\mathbf{r}) \\
1
\end{array}\right) \cdot r_{1}(\mathbf{Y}) \\
& =-\frac{1}{2 c(\mathbf{r})^{2}}\left(\rho \frac{\partial D}{\partial \rho}+\rho_{1} \frac{\partial D}{\partial \rho_{1}}+\rho_{2} \frac{\partial D}{\partial \rho_{2}}\right)+1 \\
& =-\frac{1}{2 \rho c(\mathbf{r})^{2}}\left(\alpha_{1}(\mathbf{r}) \rho_{1}^{2} p^{\prime \prime}\left(\rho_{1}\right)+\alpha_{2}(\mathbf{r}) \rho_{2}^{2} p^{\prime \prime}\left(\rho_{2}\right)\right)+1 .
\end{aligned}
$$

The densities are assumed to be strictly positive. Under the hypothesis $\left(H_{1}\right)$ and Equation (4.10) the mass fractions $\alpha_{i}$ are positive and the second derivative of the van der Waals pressure (2.11) is a strictly negative function of the density. Thus $\nabla_{\mathbf{Y}} \lambda_{1}(\mathbf{Y}) \cdot r_{1}(\mathbf{Y}) \neq 0$. Similarly we can state that $\nabla_{\mathbf{Y}} \lambda_{4}(\mathbf{Y}) \cdot r_{4}(\mathbf{Y}) \neq 0$ that conclude the proof.

We now study the wave associated to the speed $u$.

Proposition 4.4. The characteristic fields associated to the waves $\lambda_{2}(\mathbf{Y})=\lambda_{3}(\mathbf{Y})=u$ linearly degenerate i.e. $\nabla_{\mathbf{Y}} \lambda_{2}(\mathbf{Y}) \cdot r_{2}(\mathbf{Y})=0$ and $\nabla_{\mathbf{Y}} \lambda_{3}(\mathbf{Y}) \cdot r_{3}(\mathbf{Y})=0$ for admissible state vector $\mathbf{Y}$ that is for densities $\left(\rho, \rho_{1}, \rho_{2}\right)$ satisfying Equation (4.10).

Proof. We deduce from the eigenvalues (4.9) the relation

$$
\nabla_{\mathbf{Y}} \lambda_{i}(\mathbf{Y}) \cdot r_{i}(\mathbf{Y})=(0,0,0,1)^{T} \cdot r_{i}(\mathbf{Y})
$$

for $i=\{1,2\}$. Then introducing the right eigenvectors (4.11) in Equation (4.15), it is easily checked that $\nabla_{\mathbf{Y}} \lambda_{i}(\mathbf{Y}) \cdot r_{i}(\mathbf{Y})=0$ for $i=\{1,2\}$ and this complete the proof.

We now address the determination of the Riemann invariants of the system. These computations are made easier using the following property.

Proposition 4.5. The mass and volume fractions $\alpha_{1}$ and $\varphi_{1}$, defined by Equations (2.17) and (2.18), satisfy the following nonconservative equations

$$
\begin{aligned}
& \partial_{t} \alpha_{1}+u \partial_{x} \alpha_{1}=0 \\
& \partial_{t} \varphi_{1}+u \partial_{x} \varphi_{1}=0
\end{aligned}
$$

Proposition 4.6. The Riemann invariants associated to the wave of speed $u$ are

$$
\{u, \bar{p}\}
$$

with $\bar{p}(\mathbf{r})=\alpha_{1}(\mathbf{r}) p\left(\rho_{1}\right)+\alpha_{2}(\mathbf{r}) p\left(\rho_{2}\right)$. The volume and mass fractions are Riemann invariants associated to the wave of speed $u \pm c$

$$
\left\{\alpha_{1}, \varphi_{1}\right\}
$$


Proof. Because the field associated to the speed $u$ is linearly degenerate, $u$ is clearly a Riemann invariant for this wave. Using the gradient of $\bar{p}$ with respect to $\mathbf{r}$, a straightforward computation gives

$$
\nabla_{\mathbf{Y}} \bar{p}(\mathbf{Y}) \cdot r_{2}(\mathbf{Y})=0
$$

On the other hand the volume fractions $\alpha_{i}$ and the mass fractions $\varphi_{i}$ satisfy Equation (4.16). Thus the fractions are Riemann invariants for the waves of speed $u \pm c$.

The characterization of the third Riemann invariant for the waves of speed $u \pm c$ is more intricate and is not addressed here.

4.4. Invariant domains of hyperbolicity for the relaxed system. According to Section 4.2, it is clear that the homogeneous system (4.1) is hyperbolic if and only if the densities $\rho, \rho_{1}$, and $\rho_{2}$ satisfy the hypothesis $\left(H_{1}\right)$ and the constraint (4.10) on the speed of sound. Nonetheless we are interested in the study and the numerical approximation of the whole relaxed system (4.1), that is taking into account the relaxation term with a finite relaxation parameter $\varepsilon>0$. Actually the domains of hyperbolicity of the system (4.1) strongly depend on the attraction basins of the dynamical system (3.7). In the present section, we introduce the notion of invariant domains in the same spirit as in [9] for diffusive systems. We show that invariant domains $\Omega$ of hyperbolicity for the relaxed system (4.1) are subsets of the attraction basins of the dynamical system (3.7). First note that the hyperbolicity of the homogeneous system (4.1) solely depends on the densities $\mathbf{r}(t, x)=\left(\rho, \rho_{1}, \rho_{2}\right)^{t}(t, x), \forall(t, x) \in \mathbb{R}^{+} \times \mathbb{R}$, according to the constraint (4.10) on the speed of sound and not on the velocity $u(t, x)$. Hence we consider the following definition of an invariant region.

DeFinition 4.1. Let $\Omega=\left\{\mathbf{r}=\left(\rho, \rho_{1}, \rho_{2}\right) \in\right] 0,3\left[{ }^{3} \mid 0<\rho_{1} \leq \rho \leq \rho_{2}\right.$ and $\left.\rho_{1}<\rho_{2}\right\}$ a subset of the phase space $\left(\rho, \rho_{1}, \rho_{2}\right)$ with a Lipschitz continuous boundary $\partial \Omega$. The region $\Omega$ is said to be a invariant domain if

$$
\{\forall x \in \mathbb{R}, \mathbf{r}(0, x) \in \Omega\} \Leftrightarrow \forall t>0,\{\forall x \in \mathbb{R}, \mathbf{r}(t, x) \in \Omega\} .
$$

We now define some kind of indicator function for such a domain $\Omega$ : let $S$ be defined by

$$
\begin{aligned}
& S: \quad] 0,3\left[^{3} \rightarrow \mathbb{R}\right. \\
& \mathbf{r}=\left(\rho, \rho_{1}, \rho_{2}\right) \mapsto \rho s\left(\rho_{1} / \rho, \rho_{2} / \rho\right),
\end{aligned}
$$

where $s\left(\beta_{1}, \beta_{2}\right)=1-\mathbf{1}_{\left\{\beta_{i}^{-} \leq \beta_{i} \leq \beta_{i}^{+}\right\}}$. Obviously we have

$$
S(\mathbf{r})=0 \Leftrightarrow \mathbf{r} \in \Omega .
$$

Next we introduce the nonnegative quantity $J$

$$
\begin{aligned}
J: \mathbb{R}^{+} & \rightarrow \mathbb{R} \\
t & \mapsto \int_{\mathbb{R}} S(r(t, x)) d x .
\end{aligned}
$$

Proposition 4.7. Consider $\Omega$ a subset of the phase space $\left(\rho, \rho_{1}, \rho_{2}\right)$ with a Lipschitz continuous boundary $\partial \Omega$ and the associated function $S$ defined by Equation (4.20). Then one has the following properties: 
1. In the sense of distributions we have

$$
\langle\nabla S, \phi\rangle=[S] \int_{\partial \Omega} \mathbf{n} \phi(\sigma) d \sigma
$$

where $d \sigma$ is the surface measure on $\partial \Omega, \mathbf{n}$ the outer normal of $\Omega$ and $[S]=S_{\text {out }}-S_{\text {in }}$ is the jump of $S$ across the boundary $\partial \Omega$.

2. The function $S$ is positively homogeneous of degree 1 so that it verifies the Euler relation $S(\mathbf{r})=\nabla_{\mathbf{r}} S(\mathbf{r}) \cdot \mathbf{r}$.

3. The function $S$ satisfies

$$
\left\langle\nabla_{\mathbf{r}} S(\mathbf{r}), \partial_{t} \mathbf{r}+\partial_{x}(u \mathbf{r})\right\rangle=\partial_{t} S(\mathbf{r})+\partial_{x}(u S(\mathbf{r})) .
$$

Proof. The first item is a consequence of the Stokes theorem. By construction the function $S$ is positively homogeneous of degree 1. Then it satisfies the Euler relation given in the second item. Finally following the same steps as in the energy estimate (4.4), we have

$$
\begin{aligned}
& \nabla_{\mathbf{r}} S(\mathbf{r}) \partial_{t} \mathbf{r}+\nabla_{\mathbf{r}} S(\mathbf{r}) \partial_{x}(u \mathbf{r}) \\
= & \partial_{t} S(\mathbf{r})+u \partial_{x} S(\mathbf{r})+\nabla_{\mathbf{r}} S(\mathbf{r}) \cdot \mathbf{r} \partial_{x} u \\
= & \partial_{t} S(\mathbf{r})+\partial_{x}(u S(\mathbf{r})),
\end{aligned}
$$

where we use the above Euler relation for $S$ to obtain the last equality.

We now relate the definition of an invariant domain to the functions $S$ and $J$ through several propositions.

Proposition 4.8. The domain $\Omega$ is an invariant region if and only if

$$
\{J(0)=0 \Rightarrow \forall t>0, \quad J(t)=0\} .
$$

The proof of the Proposition 4.8 relies on the following Lemma.

Lemma 4.1. Let $\Omega$ be a subset of the phase space $\left(\rho, \rho_{1}, \rho_{2}\right)$ and $S$ defined by $(4.20)$. Then one has

$$
\forall x \in \mathbb{R}, \quad \mathbf{r}(., x) \in \Omega \Leftrightarrow J(.)=\int_{\mathbb{R}} S(\mathbf{r}(., x)) d x=0 .
$$

The proof of the Lemma relies on the definition of $S$ and its positivity.

Proof. (Proof of Proposition 4.8.) According to the Lemma 4.1 and by the definition of $J$ in Equation (4.21) of the quantity $J$, it follows

$$
\forall x \in \mathbb{R}, \quad r(0, x) \in \Omega \Leftrightarrow J(0)=0 .
$$

Using the Lemma 4.1 again, one gets

$$
\forall x \in \mathbb{R}, \quad r(t, x) \in \Omega \Leftrightarrow J(t)=0 .
$$

Combining these two equivalences leads to the conclusion.

Proposition 4.9. Let $\Omega$ be a subset of the phase space $\left(\rho, \rho_{1}, \rho_{2}\right)$ and $J$ given by Equation (4.21). Assume $J$ is differentiable. Then it follows

$$
\left\{\frac{d}{d t} J(t) \leq 0\right\} \Rightarrow \Omega \text { is an invariant domain }
$$


Proof. Assume that $J(0)=0$. By assumption on the time derivative of $J, J(t) \leq$ $J(0)$ and $J(t) \geq 0$ by positivity. Thus $J(t)=0, \forall t$. Hence according to Proposition 4.8, the domain $\Omega$ is invariant.

Corollary 4.1. Let $\Omega$ be a subset of the plane $\left(\rho, \rho_{1}, \rho_{2}\right)$ and $S$ and $J$ the associated functions given by Equations (4.21) and (4.20). Denote

$$
Q=\frac{1}{\varepsilon}\left(0,-\left(\rho-\rho_{1}\right)\left(\rho-\rho_{2}\right) f\left(\rho_{2} \mid \rho_{1}\right),\left(\rho-\rho_{1}\right)\left(\rho-\rho_{2}\right) f\left(\rho_{1} \mid \rho_{2}\right)\right)^{t}
$$

the right-hand side of the relaxed model (4.1). Then for any $\mathbf{r} \in \Omega$ such that

$$
\lim _{x \rightarrow+\infty} \mathbf{r}(., x)=\lim _{x \rightarrow-\infty} \mathbf{r}(., x),
$$

one has the following assertions

$$
\left\langle Q, \nabla_{\mathbf{r}} S(\mathbf{r})\right\rangle \leq 0 \Rightarrow \frac{d}{d t} J(t) \leq 0 \quad \Rightarrow \quad \Omega \text { is an invariant domain. }
$$

Proof. Since $Q$ is the right-hand side of the relaxed model (4.1), this yields

$$
\left\langle Q, \nabla_{\mathbf{r}} S(\mathbf{r})\right\rangle=\left\langle\partial_{t} \mathbf{r}+\partial_{x}(u \mathbf{r}), \nabla_{\mathbf{r}} S(\mathbf{r})\right\rangle .
$$

According to Equation (4.23) it follows that if $\left\langle Q, \nabla_{\mathbf{r}} S(\mathbf{r})\right\rangle \leq 0$ then

$$
\left\langle\partial_{t} \mathbf{r}+\partial_{x}(u \mathbf{r}), \nabla_{\mathbf{r}} S(\mathbf{r})\right\rangle \leq 0 .
$$

Integrating the above inequality on $\mathbb{R}$ gives $\int_{R} \partial_{t} S(\mathbf{r}) d x \leq 0$, that is

$$
\frac{d}{d t} \int_{R} S(\mathbf{r}) d x=\frac{d}{d t} J(t) \leq 0 .
$$

Proposition 4.9 now leads to the conclusion.

Hence in order to check that $\Omega$ is an invariant domain, one has solely to verify that

$$
\left\langle Q, \nabla_{\mathbf{r}} S(\mathbf{r})\right\rangle \leq 0 .
$$

Taking the scalar product of $\nabla S$ with the right-hand side $Q$ of the relaxation system we obtain

$$
\left\langle Q, \nabla_{\mathbf{r}} S(\mathbf{r})\right\rangle=\frac{1}{\varepsilon}\left(\rho-\rho_{1}\right)\left(\rho-\rho_{2}\right)\left(-\partial_{\rho_{1}} S f\left(\rho_{2} \mid \rho_{1}\right)+\partial_{\rho_{2}} S f\left(\rho_{1} \mid \rho_{2}\right)\right) .
$$

Using Equation (4.22), we obtain formally

$$
\left\langle Q, \nabla_{\mathbf{r}} S(\mathbf{r})\right\rangle=\frac{1}{\varepsilon}\left(\rho-\rho_{1}\right)\left(\rho-\rho_{2}\right) \rho\left(-n_{1} f\left(\rho_{2} \mid \rho_{1}\right)+n_{2} f\left(\rho_{1} \mid \rho_{2}\right)\right),
$$

where $\mathbf{n}_{\rho}=\left(n_{1}, n_{2}\right)$ is the outer normal of the domain $\Omega_{\rho}=\left\{\left(\rho_{1}, \rho_{2}\right) \mid\left(\rho, \rho_{1}, \rho_{2}\right) \in \Omega\right\}$. Note that the domain $\Omega_{\rho}$ are rectangles in the phase space $\left(\rho_{1}, \rho_{2}\right)$. Now checking the sign of $\left\langle Q, \nabla_{\mathbf{r}} S(\mathbf{r})\right\rangle$ is quite straightforward on each part of the boundary $\partial \Omega_{\rho}$. To characterize the invariant regions we study the sign of the relative Helmholtz free-energy $f(. \mid$.) defined by Equation (3.5). According to Figure 4.1, one can determine the sign of $f(. \mid$.) according to the following proposition. 


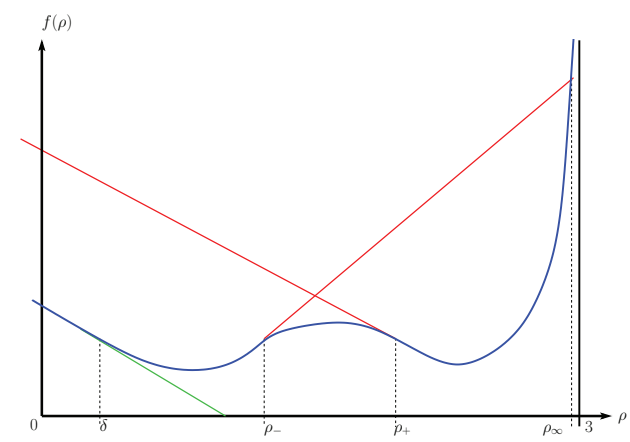

FIG. 4.1. Some reference points on the graph of the Helmholtz energy $f(\rho)$. The blue curve is a sketch of the graph of $f(\rho)$. The green line is the tangent to the graph of $f$ at the point $\delta$. The red lines are the tangents of the blue curve at $\rho^{-}$and $\rho^{+}$. The dashed line is the tangent of the graph of $f$ at $\rho_{\infty}$ defined by $f\left(\rho_{\infty}, \rho^{-}\right)=0$. Depending on the position of the tangent line to the blue curve at a given point $\rho$, one can determine the sign of $f(. \mid \rho)$, see Proposition 4.10 .

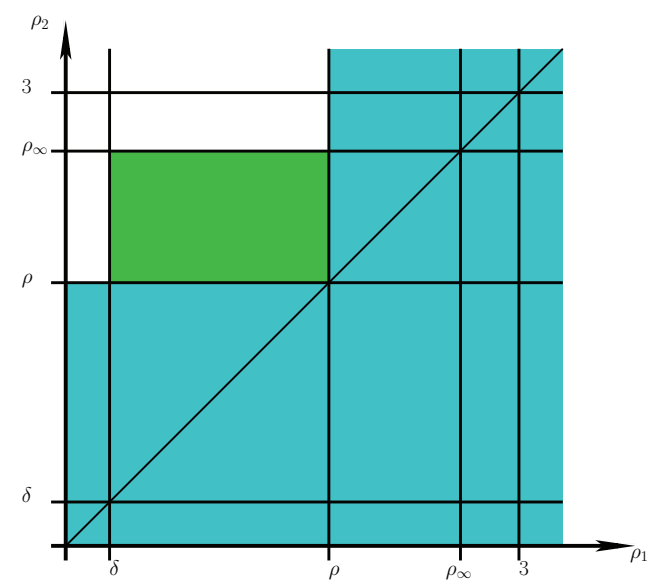

FIG. 4.2. A priori estimate for $\left(\rho_{1}, \rho_{2}\right)$ : the blue area refers to unattainable states according to $\left(H_{1}\right)$, the green zone is an invariant domain, providing in particular that void cannot appear.

Proposition 4.10. Let $\delta \in] 0, \rho_{1}^{*}\left[\right.$ and $\rho_{\infty} \in\left[\rho_{2}^{*}, 3\left[\right.\right.$ such that $f\left(\rho_{\infty} \mid \rho^{-}\right)=0$. Then the relative Helmholtz free energy satisfies

- $f(\rho \mid \delta) \geq 0, \forall \rho \in] \delta, \rho_{\infty}[$,

- $f\left(\rho \mid \rho^{-}\right) \geq 0$ (resp. $\left.\left.\leq 0\right), \forall \rho \in\right] \delta, \rho^{-}[$(resp. $\rho \in] \rho^{-}, \rho_{\infty}[$ ),

- $f\left(\rho \mid \rho^{+}\right) \leq 0$ (resp. $\left.\left.\geq 0\right), \forall \rho \in\right] \delta, \rho^{+}[$(resp. $\rho \in] \rho^{+}, \rho_{\infty}[$ ),

- $\left.f\left(\rho \mid \rho_{\infty}\right) \geq 0, \forall \rho \in\right] \delta, \rho_{\infty}[$.

Proof. The sign of $f(. \mid a)$ for any remarkable density a depends on the positition of the tangent to the graph of $f$ at the point $a$, see Figure 4.1. If the tangent at the point $a$ is below the curve (resp. above), one has $f(. \mid a)=f()-.f(a)-f^{\prime}(a)(.-a) \geq 0$ $($ resp. $\leq 0)$.

We first state a global a priori estimate which ensures that if the hypothesis $\left(H_{1}\right)$ is satisfied at $t=0$ then it is preserved for any time by the relaxed system (4.1). 
Proposition 4.11. Let $\left.\delta \in] 0, \rho_{1}^{*}\right]$ and $\rho_{\infty} \in\left[\rho_{2}^{*}, 3[\right.$ (see Figure 4.1). Then, for any $0<\rho<3$, the domain

$$
\Omega_{\rho}:=\left\{\left(\rho_{1}, \rho_{2}\right) \in(\delta, \rho) \times\left(\rho, \rho_{\infty}\right)\right\}
$$

is invariant.

Proof. One has to check the sign of $\left\langle Q, \nabla_{\mathbf{r}} S(\mathbf{r})\right\rangle$ on each side of the green rectangle domain, see Figure 4.2. On the sides $\left\{\rho_{1}=\rho\right\}$ and $\left\{\rho_{2}=\rho\right\}$ of the rectangle, $Q$ vanishes. Now for the side $\left\{\rho_{1}=\delta\right\}$, the outer normal is $\mathbf{n}_{\rho}=(-1,0)$ and

$$
\left\langle Q, \nabla_{\mathbf{r}} S(\mathbf{r})\right\rangle=\frac{1}{\varepsilon}(\rho-\delta)\left(\rho-\rho_{2}\right) \rho f\left(\rho_{2} \mid \rho^{-}\right) .
$$

The product $(\rho-\delta)\left(\rho-\rho_{2}\right) \rho$ is nonpositive and

$$
f\left(\rho_{2} \mid \rho^{-}\right)=f\left(\rho_{2}\right)-f\left(\rho^{-}\right)-f^{\prime}\left(\rho^{-}\right)\left(\rho_{2}-\rho^{-}\right) \geq 0,
$$

for $\rho_{2}>\rho_{2}^{*}$, see the green tangent line on Figure 4.1. A similar argument involving $f\left(\rho_{1} \mid \rho_{\infty}\right)$ works for the side $\left\{\rho_{2}=\rho_{\infty}\right\}$.

We turn now to determine the invariant domains of hyperbolicity of the relaxed model (4.1) depending on the value of the density $\rho$.

Proposition 4.12. Fix $0<\delta<\rho_{1}^{*}$ and let $\rho_{\infty}$ be such that $f\left(\rho_{\infty} \mid \rho^{-}\right)=0$. The following subsets $\Omega_{\rho}$ are invariant domains of hyperbolicity:

1. Pure gaseous stable zone. For any $\delta<\rho<\rho_{1}^{*}$,

$$
\Omega_{\rho}:=\left\{\left(\rho_{1}, \rho_{2}\right) \in\right] \delta, \rho^{-}[\times] \rho^{+}, \rho_{\infty}[\},
$$

see Figure 3.2.

2. Pure liquid stable zone. For any $\rho_{\infty}>\rho>\rho_{2}^{*}$,

$$
\Omega_{\rho}:=\left\{\left(\rho_{1}, \rho_{2}\right) \in\right] \rho^{+}, \rho[\times] \rho, \rho_{\infty}[\} .
$$

3. Metastable zones. For any $\delta<\rho<\rho_{\infty}$,

$$
\Omega_{\rho}:=\left\{\left(\rho_{1}, \rho_{2}\right) \in\right] \delta, \min \left(\rho, \rho^{-}\right)[\times] \max \left(\rho, \rho^{+}\right), \rho_{\infty}[\},
$$

see Figure 3.3 for the metastable vapor zone.

4. Spinodal zone. For any $\rho^{-}<\rho<\rho^{+}$,

$$
\Omega_{\rho}:=\left\{\left(\rho_{1}, \rho_{2}\right) \in\right] \delta, \rho^{-}[\times] \rho^{+}, \rho_{\infty}[\},
$$

see Figure 3.1.

Proof. We only give the proof for the spinodal zone. Following the proof of Proposition 4.11 one has to check that $\left\langle Q, \nabla_{\mathbf{r}} S(\mathbf{r})\right\rangle$ on the boundary of the green domain $\Omega_{\rho}$ of Figure 3.1. On the side $\left\{\rho_{1}=\rho^{-}\right\}$, the outer normal is $\mathbf{n}_{\rho}=(1,0)^{t}$. Thus one has

$$
\left\langle Q, \nabla_{\mathbf{r}} S(\mathbf{r})\right\rangle=\frac{1}{\varepsilon}\left(\rho-\rho^{-}\right)\left(\rho-\rho_{2}\right) \rho\left(-f\left(\rho_{2} \mid \rho^{-}\right)\right) .
$$

The product $\left(\rho-\rho^{-}\right)\left(\rho-\rho_{2}\right) \rho$ is nonpositive since $\rho>0, \rho>\rho^{-}$(because $\rho$ is fixed in $\left[\rho^{-}, \rho^{+}[)\right.$and $\rho_{2}>\rho$ thanks to hypothesis $\left(H_{1}\right)$. Moreover $f\left(\rho_{2} \mid \rho^{-}\right) \leq 0$ for any $\rho_{2} \in$ 
]$\rho^{-}, \rho_{\infty}$ [ according to Proposition 4.10. Indeed the red tangent line of the blue curve of $f$ at $\rho^{-}$is above the graph of $f$. Hence $\left\langle Q, \nabla_{\mathbf{r}} S(\mathbf{r})\right\rangle \leq 0$ on the side $\left\{\rho_{1}=\rho^{-}\right\}$. The same kind of arguments are used to prove that $\left\langle Q, \nabla_{\mathbf{r}} S(\mathbf{r})\right\rangle \leq 0$ on the three other sides of $\Omega_{\rho}$.

Note that this characterization of invariant domains of hyperbolicity is formal since it relies on the smoothness of the densities $\mathbf{r}$. A possible way to generalize to weak solutions is to follow the definition of Hoff [20].

\section{Numerical approximation}

This section is devoted to numerical experiments. We do not wish to elaborate here on efficient numerical schemes for this problem, but merely to illustrate some typical behaviours of the model. Hence we limit ourselves to a simple finite volume scheme, coupled to a time-splitting method for the source terms. Considering the stiffness of the problem, a complete numerical study is mandatory but far beyond the aim of this paper. We emphasize that we did not implement any specific strategy for the non hyperbolicity of the homogeneous system. However for all the cases we present, the computed sound velocity is real. This does not prevent from possible losses of hyperbolicity, probably due to lack of convergence in the source term treatment (see in particular sections 5.2.3 and 5.2.4).

5.1. Definition of the splitting strategy. We rewrite the system (4.1) in a more compact form, considering the following Cauchy problem

$$
\begin{aligned}
& \partial_{t} W+\partial_{x} F(W)=S(W), \\
& W(t=0, x)=W_{0}(x), \forall x \in \mathbb{R},
\end{aligned}
$$

where

$$
\begin{aligned}
W & =\left(\rho, \rho_{1}, \rho_{2}, \rho u\right)^{T}, \\
F(W) & =\left(\rho u, \rho_{1} u, \rho_{2} u, \rho u^{2}+\alpha_{1} p\left(\rho_{1}\right)+\alpha_{2} p\left(\rho_{2}\right)\right)^{T}, \\
S(W) & =\frac{1}{\varepsilon}\left(0,-\left(\rho-\rho_{1}\right)\left(\rho-\rho_{2}\right) f\left(\rho_{2} \mid \rho_{1}\right),\left(\rho-\rho_{1}\right)\left(\rho-\rho_{2}\right) f\left(\rho_{1} \mid \rho_{2}\right), 0\right),
\end{aligned}
$$

and $\varepsilon$ is the relaxation parameter. Note that we exclude pure phase initial data, so that the equations on the partial densities are not multiplied by $\alpha_{i}$ anymore.

Convective terms and source terms are taken into account by a fractional step approach. We denote $\Delta t$ the time step and $\Delta x$ the length of the cell $\left(x_{i-1 / 2}, x_{i+1 / 2}\right)$ on the regular mesh. Let $W^{n}$ be the finite volume approximation at time $t^{n}=n \Delta t, n \in \mathbb{N}$. The approximated solution $W^{n+1}$ of the Cauchy problem

$$
\left\{\begin{array}{l}
\partial_{t} W+\partial_{x} F(W)=S(W), t \in\left(t^{n}, t^{n+1}\right), x \in \mathbb{R} \\
W\left(t^{n}, x\right)=W^{n}(x), \forall x \in \mathbb{R}
\end{array}\right.
$$

is approximated by splitting the problem in two steps. The first one corresponds to the convective part

$$
\left\{\begin{array}{l}
\partial_{t} W+\partial_{x} F(W)=0, t \in\left(t^{n}, t^{n+1}\right), x \in \mathbb{R} \\
W\left(t^{n}, x\right)=W^{n}(x), \forall x \in \mathbb{R}
\end{array}\right.
$$

which provides $W^{n,-}$. The second steps corresponds to the relaxation process

$$
\left\{\begin{array}{l}
\partial_{t} W=S(W), t \in\left(t^{n}, t^{n+1}\right), x \in \mathbb{R} \\
W\left(t^{n}, x\right)=W^{n,-}(x), \forall x \in \mathbb{R}
\end{array}\right.
$$


which finally gives $W^{n+1}$.
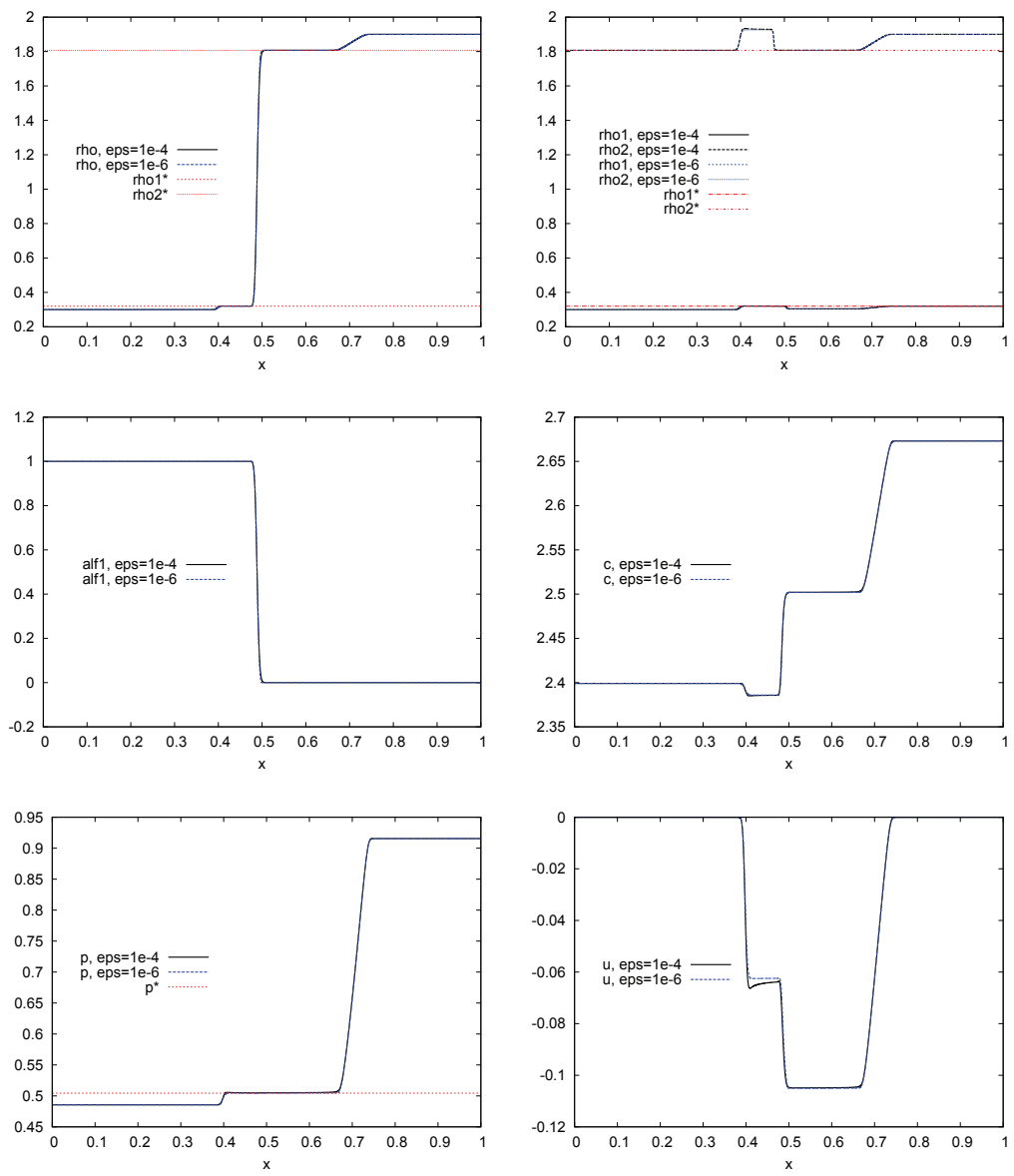

FIG. 5.1. Riemann problem with phase transition. From top left to bottom right: density $\rho$, densities $\rho_{1}$ and $\rho_{2}$, volume fraction $\alpha_{1}$, speed of sound $c$, pressure $p$, and velocity $u$.

Numerical scheme for the convective part. We consider a classical HLL numerical flux. We adopt the following classical notation:

$$
W_{i}^{n}=\frac{1}{\Delta x} \int_{x_{i-1 / 2}}^{x_{i+1 / 2}} W\left(t^{n}, x\right) d x, n \geq 0, i \in \mathbb{R} .
$$

The scheme is the following:

$$
\Delta x\left(W_{i}^{n,-}-W_{i}^{n}\right)+\Delta t\left(F_{i+1 / 2}^{n}-F_{i-1 / 2}^{n}\right)=0,
$$

together with

$$
F_{i+1 / 2}^{n}= \begin{cases}F\left(W_{i}^{n}\right), & \text { if } 0 \leq s_{L}, \\ \frac{s_{R} F\left(W_{i}^{n}\right)-s_{L} F\left(W_{i+1}^{n}\right)+s_{L} s_{R}\left(W_{i+1}^{n}-W_{i}^{n}\right)}{s_{R}-s_{L}}, & \text { if } s_{L} \leq 0 \leq s_{R} \\ F\left(W_{i+1}^{n}\right), & \text { if } 0 \geq s_{R}\end{cases}
$$



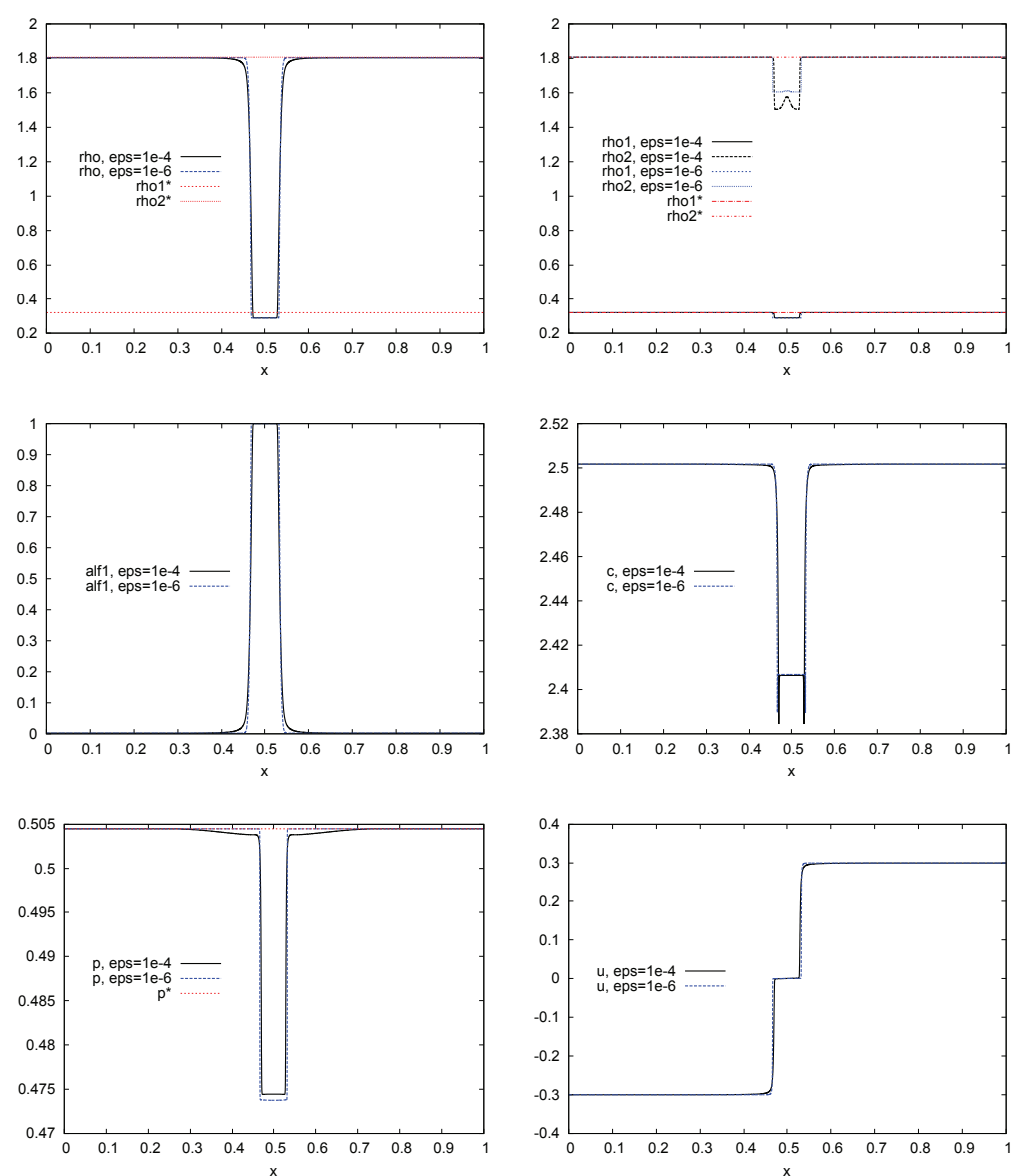

FIG. 5.2. Cavitation by double rarefaction. From top left to bottom right: density $\rho$, densities $\rho_{1}$ and $\rho_{2}$, volume fraction $\alpha_{1}$, speed of sound $c$, pressure $p$, and velocity $u$.

where $s_{R}=\max \left(u_{i}^{n}+c_{i}^{n}, u_{i+1}^{n}+c_{i+1}^{n}\right)$ and $s_{L}=\min \left(u_{i}^{n}-c_{i}^{n}, u_{i+1}^{n}-c_{i+1}^{n}\right)$. The time step is subjected to the classical CFL condition

$$
\frac{\Delta t}{\Delta x}\left|\lambda_{\max }\right| \leq 1
$$

where $\lambda_{\max }$ is the maximal speed of wave computed on each cell of the mesh.

Numerical treatment of the source terms. The initial condition for this step is $W^{n,-}$ which is assumed to be admissible, that is $\left(\rho^{n,-}, \rho_{1}^{n,-}, \rho_{2}^{n,-}\right) \in \mathcal{R}$. The total density $\rho$ and the momentum $\rho u$ remain unchanged during this step. Only the densities $\rho_{1}$ and $\rho_{2}$ may vary which leads to the following system

$$
\begin{aligned}
\partial_{t} \rho & =\partial_{t}(\rho u)=0, \\
\partial_{t} \rho_{1} & =-\frac{1}{\varepsilon}\left(\rho-\rho_{1}\right)\left(\rho-\rho_{2}\right) f\left(\rho_{2} \mid \rho_{1}\right), \\
\partial_{t} \rho_{2} & =\frac{1}{\varepsilon}\left(\rho-\rho_{1}\right)\left(\rho-\rho_{2}\right) f\left(\rho_{1} \mid \rho_{2}\right) .
\end{aligned}
$$


At this stage we merely use a classic explicit order 4 Runge-Kutta method to integrate the source term.
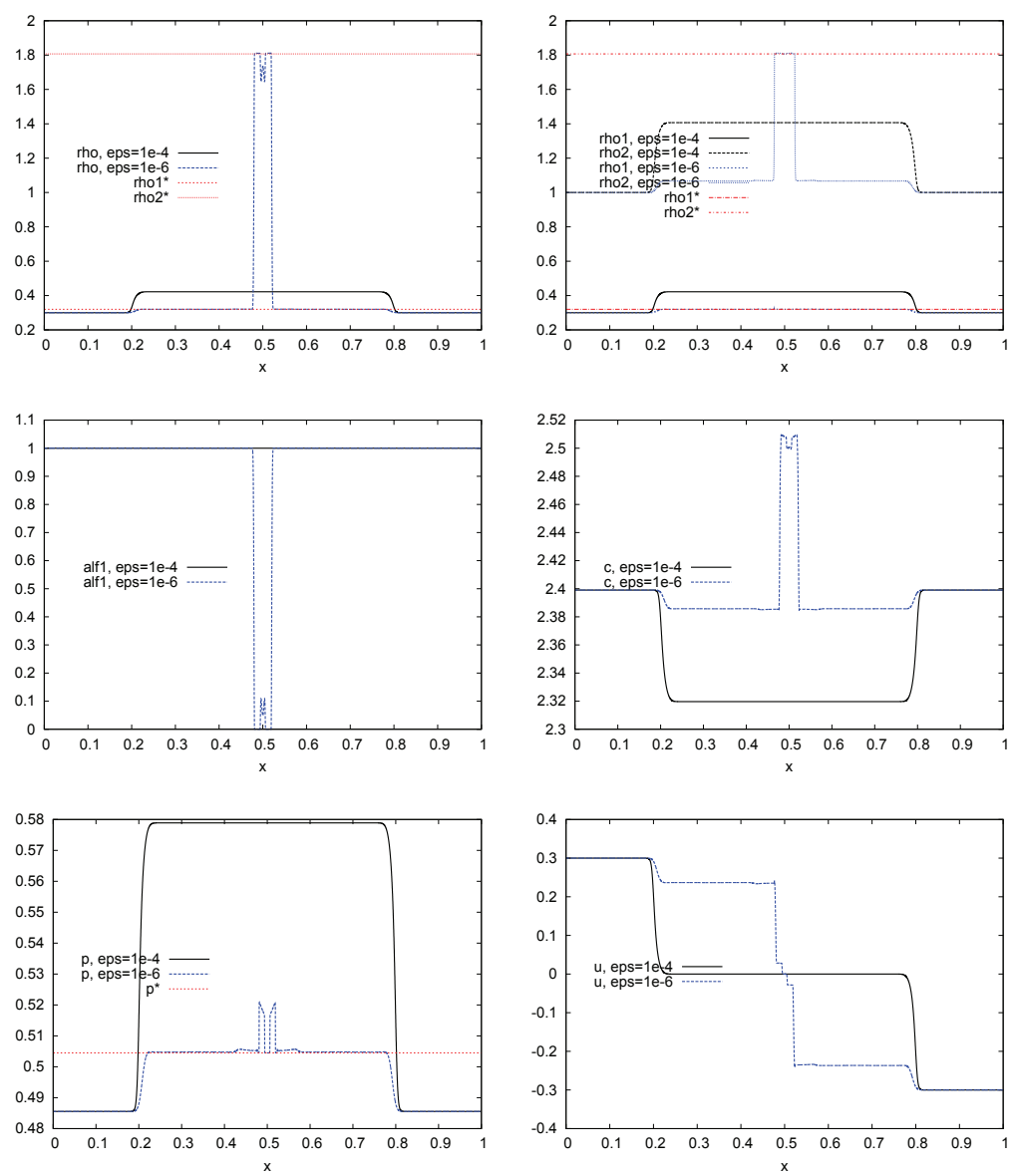

Fig. 5.3. Nucleation by double shock. From top left to bottom right: density $\rho$, densities $\rho_{1}$ and $\rho_{2}$, volume fraction $\alpha_{1}$, speed of sound $c$, pressure $p$, and velocity $u$.

Such a treatment enforces tough constraints on the time step: the computations were performed with 1000 iterations using a time step of $10^{-6}$. We emphasize that this does not ensure the actual convergence to the equilibrium state. This pleads for a more efficient method, for instance a semi-implicit scheme in the spirit of [7].

5.2. Numerical results. We present here numerical results that assess the ability of the model (4.1) to capture phase transition and metastable states.

We consider the van der Waals pressure in its reduced form (2.13) at a constant subcritical temperature $T=0.85$. At this fixed temperature the extrema $\rho^{-}$and $\rho^{+}$ of the pressure and the values $\rho_{1}^{*}$ and $\rho_{2}^{*}$ defined by the Maxwell construction on the chemical potential are given in Table 3.1.

We propose test cases with Riemann initial conditions that is

$$
W(t=0, x)=w_{0}(x)= \begin{cases}W_{L}, & \text { if } x \leq 0 \\ W_{R}, & \text { if } x>0\end{cases}
$$



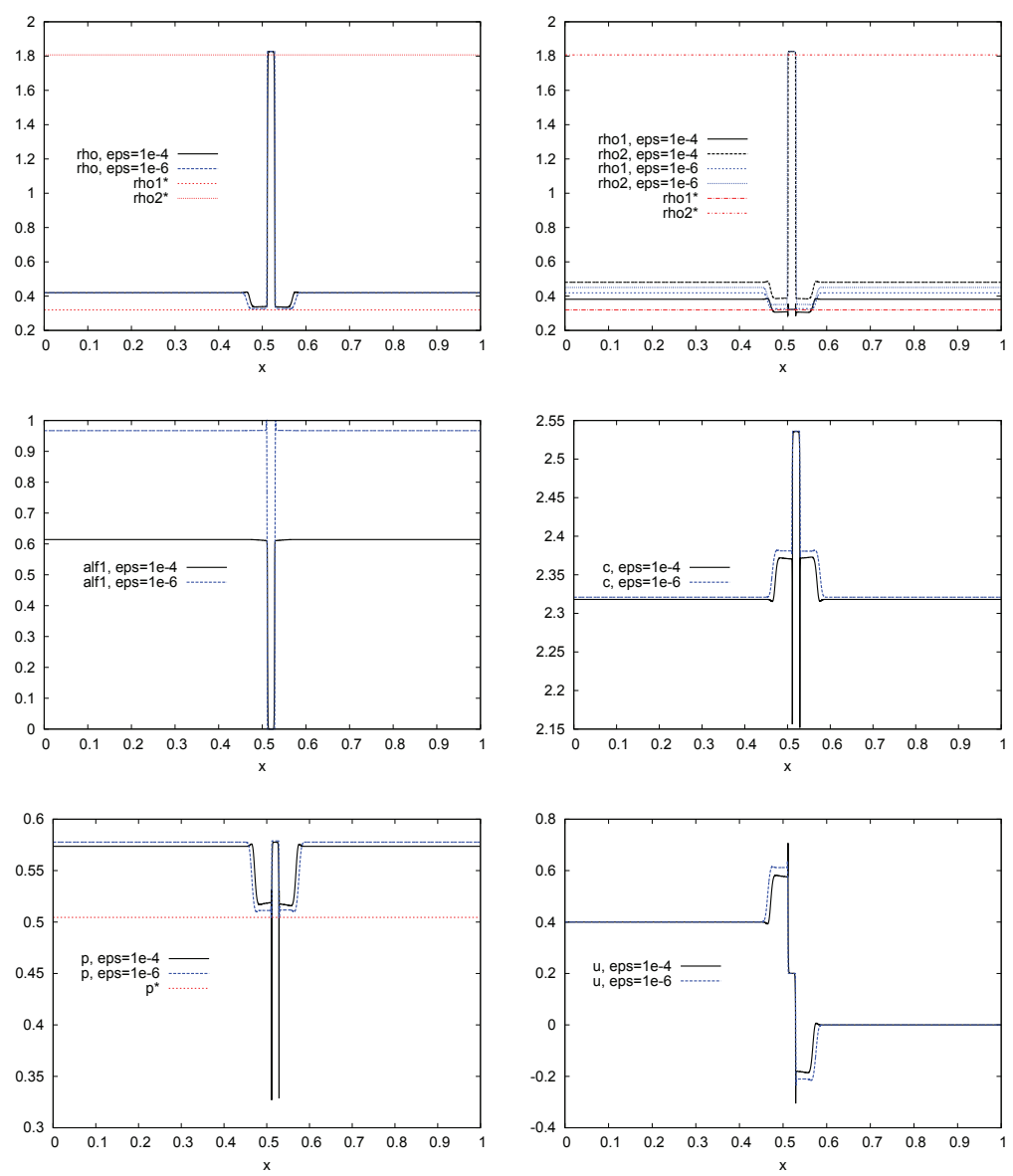

FIG. 5.4. Perturbation of a metastable state at $t=0.1 \mathrm{~s}$. From top left to bottom right: density $\rho$, densities $\rho_{1}$ and $\rho_{2}$, volume fraction $\alpha_{1}$, speed of sound $c$, pressure $p$, and velocity $u$.

The following test cases are set on the domain [0,1], with an uniform mesh of 10000 cells and Neumann boundary conditions at $x=0$ and $x=1$.

5.2.1. Riemann problem with phase transition. The initial state $W_{L}$ and $W_{R}$ are

$$
\begin{gathered}
\rho_{L}=\rho_{1, L}=0.3, \rho_{2, L}=\rho_{2}^{*}, u_{L}=0, \\
\rho_{R}=\rho_{2, R}=1.9, \rho_{1, R}=\rho_{1}^{*}, u_{R}=0 .
\end{gathered}
$$

The left state is a pure stable gas and the right state is a pure stable liquid. Various value of $\varepsilon$ are considered. The solution is at time $t=0.1 \mathrm{~s}$. One can observe the appearance of a mixture zone on both sides of the interface, see in particular the pressure profile Figure 5.1(bottom-left). The results with $\varepsilon=10^{-4}$ or $10^{-6}$ are similar expect on the velocity profile (see Figure 5.1 (bottom-right)) where the intermediate state on the left of the interface is slightly modified for $\varepsilon=10^{-4}$.

5.2.2. Cavitation by double rarefaction. The test consists in a liquid state submitted to a double rarefaction wave. The initial state is given by $\rho=\rho_{2}^{*}-10^{-3}$, 

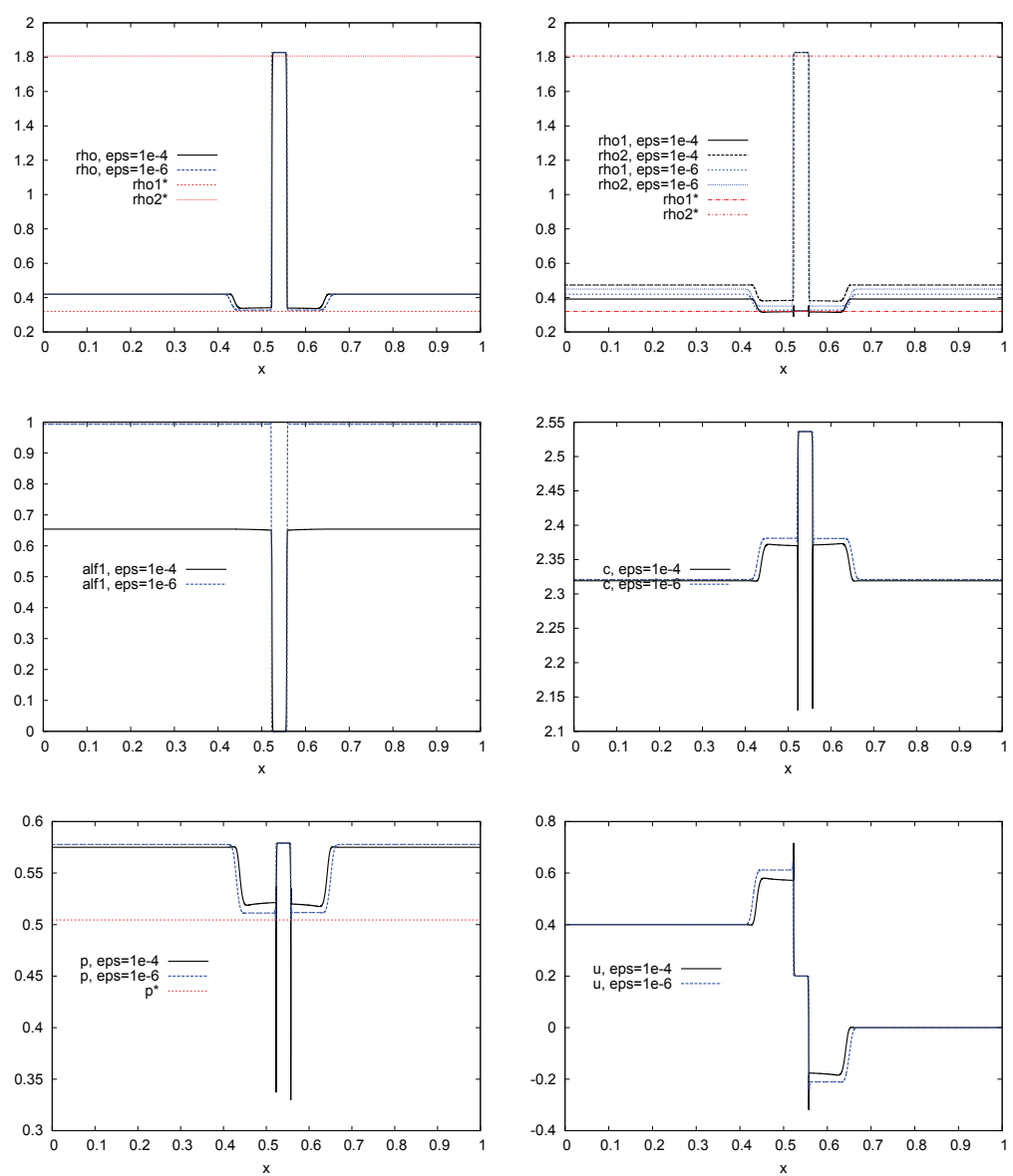

FIG. 5.5. Perturbation of a metastable state at $t=0.2 \mathrm{~s}$. From top left to bottom right: density $\rho$ densities $\rho_{1}$ and $\rho_{2}$, volume fraction $\alpha_{1}$, speed of sound $c$, pressure $p$, and velocity $u$.

$\rho_{2}=\rho_{2}^{*}, \rho_{1}=\rho_{1}^{*}$ and the velocities are $u_{R}=0.3=-u_{L}$. The total density corresponds to a metastable liquid, and the initial volume fraction is $\alpha_{1} \simeq 0.000672$, which means that phase 2 is predominant. The solution is computed at time $t=0.1 \mathrm{~s}$. We observe on the plot of the volume fraction (Figure 5.2 (middle-left)) that a bubble of stable vapor appears around the interface $x=0.5$. The value of $\varepsilon$ does not modify the profile of the bubble. However the pressure profile is sharper for $\varepsilon=10^{-6}$ than for $10^{-4}$, see Figure 5.2 (bottom-left).

5.2.3. Nucleation by double shock. The test consists in a pure stable gaseous state submitted to a double shock wave. The initial state is given by $\rho=\rho_{1}=0.3, \rho_{2}=1$ and the velocities are $u_{R}=-0.3=-u_{L}$. The solution is computed at time $t=0.4 \mathrm{~s}$. Note that phase 2 is not present initially but is fixed in the spinodal zone. We observe two different behaviours of the solution depending on the value of $\varepsilon$. For $\varepsilon=10^{-6}$, the profile of the volume fraction (see Figure 5.3 (middle-left)) shows that a droplet of liquid appears around $x=0.5$ which is not the case for $\varepsilon=10^{-4}$ where there is no droplet. For $\varepsilon=10^{-6}$, one observes on the density plot (see Figure 5.3 (top-left)) that the liquid state inside the droplet admits a density $\rho$ close to $\rho_{2}^{*}$ with small oscillations. 

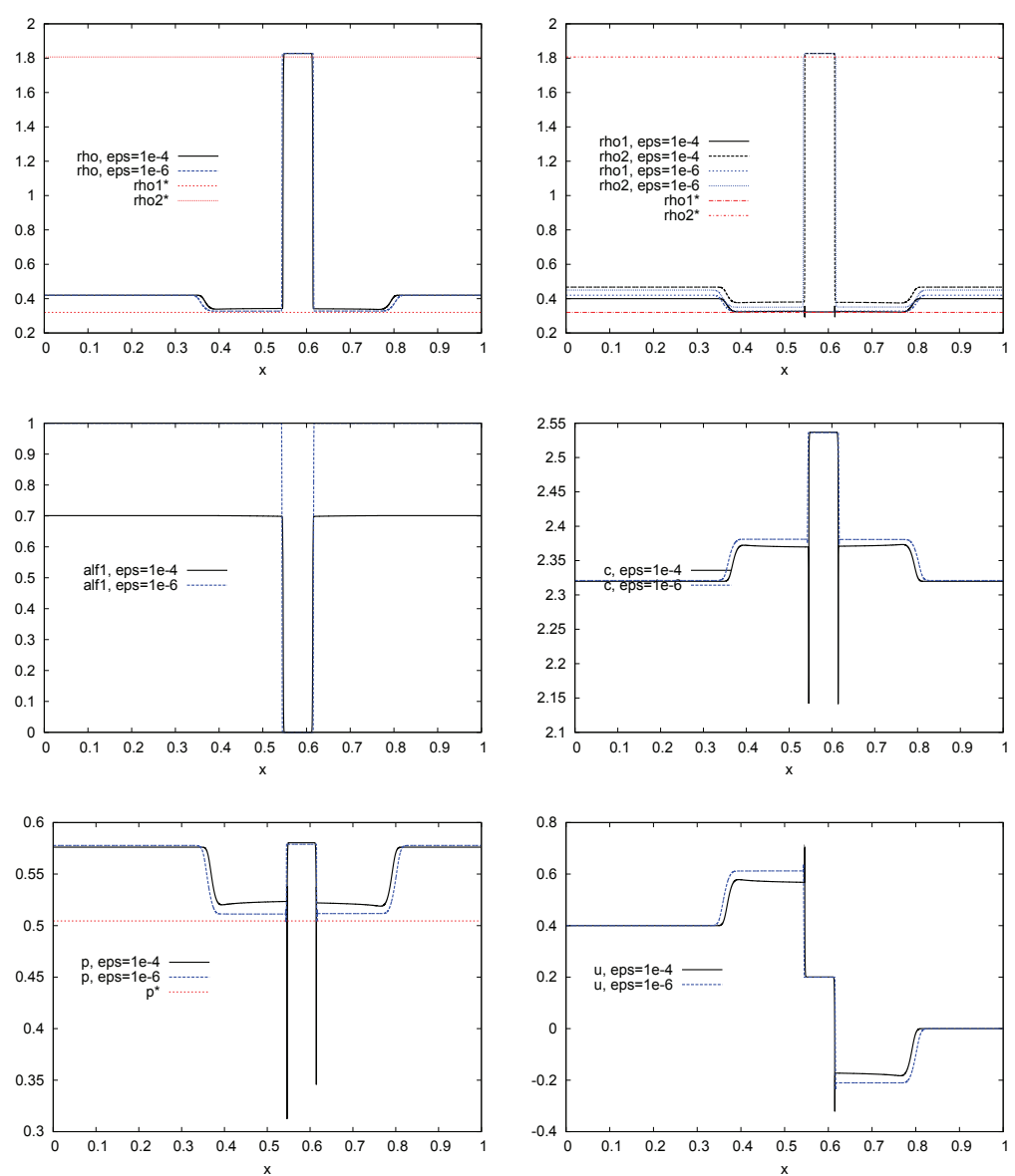

FIG. 5.6. Perturbation of a metastable state at $t=0.4 \mathrm{~s}$. From top left to bottom right: density $\rho$, densities $\rho_{1}$ and $\rho_{2}$, volume fraction $\alpha_{1}$, speed of sound $c$, pressure $p$, and velocity $u$.

The droplet is surrounded by two mixture areas with $\rho=\rho_{1}^{*}$. The pressure curve inside the droplet presents oscillations (see Figure 5.3 (bottom-left)) which might be due to a loss of hyperbolicity due to the lack of accuracy in the approximation of the source (4.21).

5.2.4. Acoustic perturbation of a metastable state. This example consists in a constant metastable vapor state, with a perturbation in the velocity. The initial state is $\rho=0.42, \rho_{1}=0.32, \rho_{2}=0.52$ and the velocities are $u_{L}=0.4, u_{R}=0$. Both densities $\rho_{1}$ and $\rho_{2}$ are in the metastable state, and we impose a compression from the left with velocity 0.4 .

The compression induces the appearance of droplet of pure liquid which moves from the left to the right, see the time evolution on Figure 5.4. With a smaller velocity perturbation the structure of the waves is similar, but there is no creation of a droplet at the interface. One can check on Figure 5.4 (top-left) that the density $\rho$ inside the droplet is larger than $\rho_{2}^{*}$. The droplet is surrounded by two areas with a mixture state with $\rho=\rho_{1}^{*}$. The velocity and pressure profiles exhibit spikes on both sides of the droplet, which amplitude decreases when $\varepsilon$ decreases, see figures 5.4, 5.5, and 5.6. Also notice 
that the pressure in the mixture zone is not at the value $p^{*}$. It seems that when $\varepsilon$ decreases the value is closer, this may indicate that the source term has not reached the equilibrium state yet.

\section{Conclusion}

The core of this work is the formalization in terms of a dynamical system of the thermodynamics of phase transition, using the van der Waals EoS. It leads to a mathematical characterization of metastable states, compared to stable coexistent two-phase states. The dynamical behaviour of the solutions is crucial here, and seems to preclude the use of instantaneous exchange kinetic. When coupled to a simple hydrodynamic model, namely the isothermal Euler equations, it evidences abilities to cope with metastable states as well as bubble or droplet generation. This preliminary study gives rise to a wide bunch of open questions and problems, and we believe that the methodology can be used in a much larger context.

First, in the same isothermal context, the construction of the dynamical system (the right-hand side in the extended Euler equations) can be addressed. We deliberately used a simple and readable function, which possibly could be improved. In any case, the behaviour of the coexistence zone around the interface has to be investigated in more details, as well as the role of $\varepsilon$.

Next, an obvious mandatory issue is the numerical treatment of the coupled system. We have chosen here the simplest numerical strategy that allowed us to illustrate our purpose. The explicit treatment of the stiff relaxation term enforces tough constraints on the time step, and prevents the simulation of more realistic metastable cases.

Finally, we attend to include temperature dependance to obtain a fully heat, mass, and mechanical transfer model in order to compare our results to those of [32] and [35].

\section{REFERENCES}

[1] R. Abeyaratne and J.K. Knowles, Kinetic relations and the propagation of phase boundaries in solids, Arch. Rat. Mech. Anal., 114(2), 119-154, 1991.

[2] G. Allaire, G. Faccanoni, and S. Kokh, A strictly hyperbolic equilibrium phase transition model, C.R. Math. Acad. Sci. Paris, 344(2), 135-140, 2007.

[3] A. Ambroso, C. Chalons, F. Coquel, and T. Galié, Relaxation and numerical approximation of a two-fluid two-pressure diphasic model, M2AN Math. Model. Numer. Anal., 43(6), 1063-1097, 2009.

[4] M.R. Baer and J.W. Nunziato, A two phase mixture theory for the deflagration to detonation (ddt) transition in reactive granular materials, Int. J. Multiphase Flow, 12(6), 861-889, 1986.

[5] T. Barberon and P. Helluy, Finite volume simulation of cavitating flows, Computers and Fluids, 34(7), 832-858, 2005.

[6] N. Bedjaoui, C. Chalons, F. Coquel, and P.G. LeFloch, Non-monotonic traveling waves in van der Waals fluids, Anal. Appl. (Singap.), 3(4), 419-446, 2005.

[7] S. Boscarino and G. Russo, On a class of uniformly accurate IMEX Runge-Kutta schemes and applications to hyperbolic systems with relaxation, SIAM J. Sci. Comput., 31(3), 1926-1945, 2009.

[8] H.B. Callen, Thermodynamics and an Introduction to Thermostatistics, Second Edition, Wiley and Sons, 1985.

[9] K. Chueh, C. Conley, and J. Smoller, Positively invariant regions for systems of nonlinear diffusion equations, Indiana Univ. Math. J., 26(2), 373-392, 1977.

[10] A. Corli and H. Fan, The Riemann problem for reversible reactive flows with metastability, SIAM J. Appl. Math., 65(2), 426-457 (electronic), 2004/05.

[11] J.-M. Delhaye, M. Giot, L. Mahias, P. Raymond, and C. Rénault, Thermohydraulique des Racteurs, EDP Sciences, 1998.

[12] J.M. Delhaye, M. Giot, and M.I. Riethmuller, Thermohydraulics of two-phase systems for industrial design and nuclear engineering, Hemisphere Publishing Corporation, 1981.

[13] D.A. Drew, Mathematical modeling of two-phase flow, Ann. Rev. Fluid Mech., 15, 261-291, 1983. 
[14] G. Faccanoni, S. Kokh, and G. Allaire, Modelling and simulation of liquid-vapor phase transition in compressible flows based on thermodynamic equilibrium, ESAIM Math. Model. Numer. Anal., 46(5), 1029-1054, 2012.

[15] H. Fan and X.-B. Lin, A dynamical systems approach to traveling wave solutions for liquid/vapor phase transition, in Infinite dimensional dynamical systems, Fields Inst. Commun., Springer, New York, 64, 101-117, 2013.

[16] J.W. Gibbs, The Collected Works of J. Willard Gibbs, vol. I: Thermodynamics, Yale University Press, 1948.

[17] P. Helluy and H. Mathis, Pressure laws and fast Legendre transform, Math. Models Meth. Appl. Sci., 21(4), 745-775, 2011.

[18] P. Helluy and N. Seguin, Relaxation models of phase transition flows, M2AN Math. Model. Numer. Anal., 40(2), 331-352, 2006.

[19] J.-B. Hiriart-Urruty and C. Lemaréchal, Fundamentals of convex analysis, Grundlehren Text Editions, Springer-Verlag, Berlin, 2001.

[20] D. Hoff, Invariant regions for systems of conservation laws, Trans. Amer. Math. Soc., 289(2), 591-610, 1985.

[21] A.R. Imre, I.F. Barna, G. Ézösl, G. Házi, and T. Kraska, Theoretical study of flashing and water hammer in a supercritical water cycle during pressure drop, Nuclear Engineering and Design, 240(6), 1569-1574, 2010.

[22] F. Jaegle, C. Rohde, and C. Zeiler, A multiscale method for compressible liquid-vapor flow with surface tension, in CEMRACS'11: Multiscale coupling of complex models in scientific computing, ESAIM Proc., EDP Sci., Les Ulis, 38, 387-408, 2012.

[23] F. James, M. Sepúlveda, and P. Valentin, Statistical thermodynamics models for multicomponent isothermal diphasic equilibria, Math. Models Meth. Appl. Sci., 7(1), 1-29, 1997.

[24] S. Jaouen, Etude mathématique et numérique de stabilité pour des modèles hydrodynamiques avec transition de phase, $\mathrm{PhD}$ thesis, Universit Paris VI, November 2001.

[25] L. Landau and E. Lifschitz, A Course of Theoretical Physics, Statistical Physics, Pergamon Press, 5, 1959-1969, 1978.

[26] S. Le Martelot, R. Saurel, and B. Nkonga, Towards the direct numerical simulation of nucleate boiling flows, Int. J. Multiph. Flow, 66, 62-78, 2014.

[27] P.G. LeFloch, Kinetic relations for undercompressive shock waves. Physical, mathematical, and numerical issues, in Nonlinear partial differential equations and hyperbolic wave phenomena, Contemp. Math., Amer. Math. Soc., Providence, RI, 526, 237-272, 2010.

[28] H. Mathis, Étude théorique et numérique des écoulements avec transition de phase, PhD thesis, Université de Strasbourg, 2010.

[29] A. Murrone and H. Guillard, A five equation reduced model for compressible two phase flow problems, J. Comput. Phys., 202(2), 664-698, 2005.

[30] M. Pelanti and K.-M. Shyue, A mixture-energy-consistent six-equation two-phase numerical model for fluids with interfaces cavitation and evaporation waves, J. Comput. Phys., 259, 331-357, 2014.

[31] R.T. Rockafellar, Convex Analysis, Princeton Landmarks in Mathematics. Princeton University Press, Princeton, NJ, Princeton Paperbacks, reprint, 1997.

[32] R. Saurel, F. Petitpas, and R. Abgrall, Modelling phase transition in metastable liquids: application to cavitating and flashing flows, J.Fluid Mech., 607, 313-350, 2008.

[33] M. Slemrod, Admissibility criteria for propagating phase boundaries in a van der Waals fluid, Arch. Rat. Mech. Anal., 81(4), 301-315, 1983.

[34] M. Slemrod, Dynamic phase transitions in a van der Waals fluid, J. Diff. Eqs., 52(1), 1-23, 1984.

[35] A. Zein, M. Hantke, and G. Warnecke, Modeling phase transition for compressible two-phase flows applied to metastable liquids, J. Comput. Phys., 229, 1964-2998, 2010. 